\title{
Article \\ Fixed Point Results for New Types of Fuzzy Contractions via Admissible Functions and $\mathcal{F} \mathcal{Z}$-Simulation Functions
}

\author{
Abdelhamid Moussaoui ${ }^{1}{ }^{(0)}$, Naeem Saleem ${ }^{2}{ }^{(\mathbb{C}}$, Said Melliani ${ }^{1}(\mathbb{D})$ and Mi Zhou ${ }^{3,4, *}$ \\ 1 Laboratory of Applied Mathematics and Scientific Computing, Faculty of Sciences and Technics, \\ Sultan Moulay Slimane University, P.O. Box 523, Beni Mellal 23000, Morocco; a.moussaoui@usms.ma (A.M.); \\ s.melliani@usms.ma (S.M.) \\ 2 Department of Mathematics, University of Management and Technology, Lahore 54770, Pakistan; \\ naeem.saleem2@gmail.com \\ 3 School of Science and Technology \& Center for Mathematical Research, University of Sanya, \\ Sanya 572000, China \\ 4 Academician Guoliang Chen Team Innovation Center \& Academician Chunming Rong Workstation, \\ University of Sanya, Sanya 572022, China \\ * Correspondence: mizhou@sanyau.edu.cn or mizhou330@126.com
}

Citation: Moussaoui, A.; Saleem, N.; Melliani, S.; Zhou, M. Fixed Point Results for New Types of Fuzzy Contractions via Admissible Functions and $\mathcal{F} \mathcal{Z}$-Simulation Functions. Axioms 2022, 11, 87. https://doi.org/10.3390/ axioms11030087

Academic Editor: Hsien-Chung Wu

Received: 17 January 2022

Accepted: 18 February 2022

Published: 23 February 2022

Publisher's Note: MDPI stays neutral with regard to jurisdictional claims in published maps and institutional affiliations.

Copyright: (C) 2022 by the authors. Licensee MDPI, Basel, Switzerland. This article is an open access article distributed under the terms and conditions of the Creative Commons Attribution (CC BY) license (https:// creativecommons.org/licenses/by/ $4.0 /)$.

\begin{abstract}
In this paper, we introduce two new concepts, generalized $\alpha-\eta-\mathcal{F} \mathcal{Z}$-contraction and modified $\alpha-\eta-\mathcal{F} \mathcal{Z}$-contraction, which unify several types of contractions in the context of fuzzy metric spaces. We discuss the existence and uniqueness results of such mappings in the setting of a complete fuzzy metric space in the sense of George and Veeramani and present several significant consequences of our obtained results by using variant examples for $\mathcal{F} \mathcal{Z}$-simulation functions and admissible mappings. Some examples are provided to illustrate the usability of our main results.
\end{abstract}

Keywords: generalized $\alpha-\eta-\mathcal{F} \mathcal{Z}$-contraction; modified $\alpha-\eta-\mathcal{F} \mathcal{Z}$-contraction; fuzzy metric spaces

\section{Introduction}

Fixed point theory is one of the most influential theories in nonlinear functional analysis. Several disciplines, including computer sciences (see [1,2]), economics, game theory [3], and many more, used fixed point techniques to find solutions of nonlinear equations. The pioneering Banach Contraction Principle is a major progress in the theoretical development of metric fixed point theory. This result has been developed, extended, generalized, and improved in several generalized abstract spaces (see [4-15]).

In 2015, Khojasteh et al. [9] provided a novel approach to prove the existence of fixed point by exploring the concept of simulation functions, which exhibit a significant unifying power. Accordingly, many researchers extended and enriched this notion in various distinct metric spaces (see [11,14,16-18]). In 2018, inspired by the aforementioned approach, Melliani and Moussaoui [8,19] proposed a new type of fuzzy contractions, called $\mathcal{F} \mathcal{Z}$-contraction in the context of fuzzy metric spaces and showed that this new form of contractions can also yield a unique point of view for various well-known concepts such as fuzzy contractions [5], fuzzy $\psi$-contractions [6], and fuzzy $\mathcal{H}$-contractions [7]. Other remarkable results were presented in [10] where the concept of admissible mappings was initiated to include numerous and diverse contractions. For more details, see $[10,20]$.

In this study, we define two new concepts of contractions named generalized $\alpha-\eta$ $\mathcal{F} \mathcal{Z}$-contraction and modified $\alpha-\eta-\mathcal{F} \mathcal{Z}$-contraction, and prove some fixed point results which ensure the existence of unique fixed point for these new contractions in complete fuzzy metric spaces in the sense of George and Veeramani. Furthermore, we derive several distinct fixed point results by properly defining the admissible mapping and / or the $\mathcal{F} \mathcal{Z}$ simulation function in our main theorems. The presented results enrich, generalize, extend, and unify various previously released results in the literature. 


\section{Preliminaries}

For reasons of comprehensiveness, we will discuss in brevity some essential notions.

Definition 1 ([21]). A continuous t-norm is a binary operator $*:[0,1] \times[0,1] \longrightarrow[0,1]$ satisfying the following four conditions:

$\left(\mathcal{C} \mathcal{T}_{1}\right) *$ is commutative and associative,

$\left(\mathcal{C T}_{2}\right) *$ is continuous,

$\left(\mathcal{C T}_{3}\right) \varsigma * 1=\varsigma$ for all $\varsigma \in[0,1]$,

$\left(\mathcal{C T}_{4}\right) \varsigma * v \leq \sigma * \pi$ whenever $\varsigma \leq \sigma$ and $v \leq \pi$, for all $\varsigma, v, \sigma, \pi \in[0,1]$.

Example 1. The following ones are classical examples of continuous t-norm:

(1) $\varsigma * v=\varsigma \cdot v$,

(2) $\varsigma * v=\max \{0, \varsigma+v-1\}$,

(3) $\varsigma * v=\min \{\varsigma, v\}$.

Definition 2 (George and Veeramani [22]). A fuzzy metric space (FMS) is an ordered 3-triple $(\mathcal{S}, \mathcal{L}, *)$ where $\mathcal{S}$ is an arbitrary nonempty set, $*$ is a continuous $t$-norm, and $\mathcal{L}$ is fuzzy set on $\mathcal{S}^{2} \times(0, \infty)$ satisfying the following conditions, for all $\theta, \vartheta, \delta \in \mathcal{S}$ and $\varsigma, s>0$,

$(\mathcal{G} \mathcal{V} 1) \mathcal{L}(\theta, \vartheta, \varsigma)>0$

$(\mathcal{G} \mathcal{V} 2) \mathcal{L}(\theta, \vartheta, \varsigma)=1$ if and only if $\theta=\vartheta$,

$(\mathcal{G} \mathcal{V} 3) \mathcal{L}(\theta, \vartheta, \varsigma)=\mathcal{L}(\vartheta, \theta, \varsigma)$

$(\mathcal{G} \mathcal{V} 4) \quad \mathcal{L}(\theta, \vartheta, \varsigma) * \mathcal{L}(\vartheta, \delta, s) \leq \mathcal{L}(\theta, \delta, \varsigma+s)$

$(\mathcal{G} \mathcal{V} 5) \mathcal{L}(\theta, \vartheta, \cdot):(0,+\infty) \rightarrow[0,1]$ is continuous.

If we replace $(\mathcal{G} \mathcal{V} 4)$ by

$$
(\mathcal{G} \mathcal{V} 4)^{\prime}: \mathcal{L}(\theta, \vartheta, \varsigma) * \mathcal{L}(\vartheta, \delta, s) \leq \mathcal{L}(\theta, \delta, \max \{\varsigma, s\})
$$

for all $\theta, \vartheta, \delta \in \mathcal{S}$ and $\varsigma, s>0$, then the ordered triple $(\mathcal{S}, \mathcal{L}, *)$ is called a non-Archimedean FMS, as $(\mathcal{G} \mathcal{V} 4)^{\prime} \Rightarrow(\mathcal{G} \mathcal{V} 4)$, every non-Archimedean FMS is a FMS. Furthermore, $(\mathcal{S}, \mathcal{L}, *)$ is called a strong FMS if $(\mathcal{G} \mathcal{V} 4)$ is changed by $(\mathcal{G} \mathcal{V} 4)^{\prime \prime}: \mathcal{L}(\theta, \vartheta, \varsigma) * \mathcal{L}(\vartheta, \delta, \varsigma) \leq \mathcal{L}(\theta, \delta, \varsigma)$ for all $\theta, \vartheta, \delta \in \mathcal{S}$ and $\varsigma>0$.

According to [22], every fuzzy metric $\mathcal{L}$ on a set $\mathcal{S}$ yields a Hausdorff first countable topology $\tau_{\mathcal{L}}$ on $\mathcal{S}$, with bases as the family of open balls $\left\{B_{\mathcal{L}}(\theta, r, \varsigma): \theta \in \mathcal{S}, r \in(0,1), \varsigma>\right.$ $0\}$, where $B_{\mathcal{L}}(\theta, r, \varsigma)=\{\vartheta \in \mathcal{S}: \mathcal{L}(\theta, \vartheta, \varsigma)>1-r\}$. For more details, see [5,22].

Lemma $1([4])$. $\mathcal{L}(\theta, \vartheta, \cdot)$ is nondecreasing on $(0,+\infty)$ for all $\theta, \vartheta$ in $\mathcal{S}$.

Definition 3 ([22]). Let $(\mathcal{S}, \mathcal{L}, *)$ be a FMS.

1. A sequence $\left\{\theta_{n}\right\} \subseteq \mathcal{S}$ is said to be convergent to $\theta \in \mathcal{S}$ if $\lim _{n \rightarrow \infty} \mathcal{L}\left(\theta_{n}, \theta, \varsigma\right)=1$ for all $\varsigma>0$;

2. A sequence $\left\{\theta_{n}\right\} \subseteq \mathcal{S}$ is said to be a Cauchy sequence if and only if for all $\varepsilon \in(0,1)$ and $\varsigma>0$, there exists $n_{0} \in \mathbb{N}$ such that $\mathcal{L}\left(\theta_{n}, \theta_{m}, \varsigma\right)>1-\varepsilon$ for all $n, m \geq n_{0}$;

3. A FMS in which every Cauchy sequence is convergent is said to be complete.

Definition 4 ([5]). Let $(\mathcal{S}, \mathcal{L}, *)$ be a FMS. A mapping $\mathcal{G}: \mathcal{S} \rightarrow \mathcal{S}$ is said to be a fuzzy contractive mapping if

$$
\frac{1}{\mathcal{L}(\mathcal{G} \theta, \mathcal{G} \vartheta, \varsigma)}-1 \leq \kappa\left(\frac{1}{\mathcal{L}(\theta, \vartheta, \varsigma)}-1\right)
$$


for all $\theta, \vartheta \in \mathcal{S}, \varsigma>0$ and some $\kappa \in(0,1)$.

Let $\Psi$ be the set of functions $\psi:[0,1] \rightarrow[0,1]$ such that $\psi$ is non-decreasing, continuous, and $\psi(\hbar)>\hbar$, for all $\hbar \in(0,1)$.

Definition 5 ([6]). Let $(\mathcal{S}, \mathcal{L}, *)$ be a FMS. A mapping $\mathcal{G}: \mathcal{S} \rightarrow \mathcal{S}$ is said to be a fuzzy $\Psi$ contractive mapping if

$$
\mathcal{L}(\mathcal{G} \theta, \mathcal{G} \vartheta, \varsigma) \geq \psi(\mathcal{L}(\theta, \vartheta, \varsigma)) \text { for all } \theta, \vartheta \in \mathcal{S}, \varsigma>0, \psi \in \Psi .
$$

Let $\mathcal{H}$ denote the class of strictly decreasing functions $\eta:(0,1] \rightarrow[0,+\infty)$ such that $\eta$ transforms $(0,1]$ onto $[0,+\infty)$.

Definition 6 (Wardowski [7]). Let $(\mathcal{S}, \mathcal{L}, *)$ be a FMS. A mapping $\mathcal{G}: \mathcal{S} \rightarrow \mathcal{S}$ is said to be a fuzzy $\mathcal{H}$-contractive with respect to $\eta \in \mathcal{H}$ if there exists $\kappa \in(0,1)$ such that

$$
\eta(\mathcal{L}(\mathcal{G} \theta, \mathcal{G} \vartheta, \varsigma)) \leq \kappa \eta(\mathcal{L}(\theta, \vartheta, \varsigma)) \text { for all } \theta, \vartheta \in \mathcal{S}, \varsigma>0
$$

On the other hand, we are interested to recall the notion of $\mathcal{F} \mathcal{Z}$-contraction, recently introduced by Melliani and Moussaoui $([8,19])$. Precisely, this concept is a new type of fuzzy contraction defined by using a specific control function, stated as follows.

Definition 7 ([8,19]). A mapping $\zeta:(0,1] \times(0,1] \rightarrow \mathbb{R}$ is said to be an $\mathcal{F} \mathcal{Z}$-simulation function if it satisfies the following conditions:

$(\zeta 1) \zeta(1,1)=0$



(उ3) if $\left\{\omega_{n}\right\},\left\{\omega_{n}\right\}$ are sequences in $(0,1]$ such that $\lim _{n \rightarrow+\infty} \omega_{n}=\lim _{n \rightarrow+\infty} \omega_{n}<1$ then $\lim _{n \rightarrow+\infty} \sup \zeta\left(\omega_{n}, \omega_{n}\right)<0$.

The collection of all $\mathcal{F} \mathcal{Z}$-simulation functions is denoted by $\mathcal{F Z}$.

Next, we give some examples of the $\mathcal{F} \mathcal{Z}$-simulation function.

Example 2 ([8,19]). Let $\zeta_{l}:(0,1] \times(0,1] \longrightarrow \mathbb{R}, l=1,2,3$ be defined as:

1. $\zeta_{1}(\omega, \omega)=\kappa\left(\frac{1}{\omega}-1\right)-\frac{1}{\omega}+1$ for all $\omega, \omega \in(0,1]$, where $\kappa \in(0,1)$;

2. $\zeta_{2}(\omega, \omega)=\frac{1}{\psi(\omega)}-\frac{1}{\omega}$ for all $\omega, \omega \in(0,1]$ and $\psi \in \Psi$;

3. $\zeta_{3}(\omega, \omega)=\frac{1}{\eta^{-1}(\kappa \cdot \eta(\omega))}-\frac{1}{\omega}$ for all $\omega, \omega \in(0,1]$, where $\eta \in \mathcal{H}$.

Then, $\zeta_{l}(l=1,2,3)$ are $\mathcal{F} \mathcal{Z}$-simulation functions.

Definition $8([8,19])$. Let $(\mathcal{S}, \mathcal{L}, *)$ be a FMS and $\zeta \in \mathcal{F} \mathcal{Z}$. A mapping $\mathcal{G}: \mathcal{S} \rightarrow \mathcal{S}$ is said to be an $\mathcal{F} \mathcal{Z}$-contraction with respect to $\zeta$, if the following condition is satisfied

$$
\zeta(\mathcal{L}(\mathcal{G} \theta, \mathcal{G} \vartheta, \varsigma), \mathcal{L}(\theta, \vartheta, \varsigma)) \geq 0 \text { for all } \theta, \vartheta \in \mathcal{S}, \varsigma>0 .
$$

Definition 9 ([23]). Let $(\mathcal{S}, \mathcal{L}, *)$ be a FMS and let $\alpha, \eta: \mathcal{S} \times \mathcal{S} \times(0,+\infty) \rightarrow[0,+\infty)$ be two functions. We say that $\mathcal{G}: \mathcal{S} \rightarrow \mathcal{S}$ is $\alpha$-admissible with respect to $\eta$ if, for all $\theta, \vartheta \in \mathcal{S}$

$$
\alpha(\theta, \vartheta, \varsigma) \geq \eta(\theta, \vartheta, \varsigma) \Rightarrow \alpha(\mathcal{G} \theta, \mathcal{G} \vartheta, \varsigma) \geq \eta(\mathcal{G} \theta, \mathcal{G} \vartheta, \varsigma) \text { for all } \varsigma>0 .
$$

if we take $\alpha(\theta, \vartheta, \varsigma)=1$ for all $\theta, \vartheta \in \mathcal{S}$ and $\varsigma>0$, then we say that $\mathcal{G}$ is an $\eta$-subadmissible mapping.

In line with [24], we use the concepts of $\alpha$-orbital and triangular $\alpha$-orbital admissibility in the following form. 
Definition 10 ([24]). Let $(\mathcal{S}, \mathcal{L}, *)$ be a FMS and let $\alpha, \eta: \mathcal{S} \times \mathcal{S} \times(0,+\infty) \rightarrow[0,+\infty)$ be two functions. We say that $\mathcal{G}: \mathcal{S} \rightarrow \mathcal{S}$ is $\alpha$-orbital admissible with respect to $\eta$ if

$$
\alpha(\theta, \mathcal{G} \theta, \varsigma) \geq \eta(\theta, \mathcal{G} \theta, \varsigma) \Longrightarrow \alpha\left(\mathcal{G} \theta, \mathcal{G}^{2} \theta, \varsigma\right) \geq \eta\left(\mathcal{G} \theta, \mathcal{G}^{2} \theta, \varsigma\right)
$$

Moreover, $\mathcal{G}$ is said to be triangular $\alpha$-orbital admissible with respect to $\eta$ if it satisfies the following conditions:

(T1) $\mathcal{G}$ is $\alpha$-orbital admissible with respect to $\eta$;

$(T 2) \alpha(\theta, \vartheta, \varsigma) \geq \eta(\theta, \vartheta, \varsigma)$ and $\alpha(\vartheta, \mathcal{G} \vartheta, \varsigma) \geq \eta(\vartheta, \mathcal{G} \vartheta, \varsigma) \Rightarrow \alpha(\theta, \mathcal{G} \vartheta, \varsigma) \geq \eta(\theta, \mathcal{G} \vartheta, \varsigma)$.

Remark 1. Note that if we take $\eta(\theta, \vartheta, \varsigma)=1$, then this definition reduces to the definition of $\alpha$-orbital and triangular $\alpha$-orbital admissible mappings.

In 2016, Mishra et al. [23] initiated the idea of a modified $\alpha-\phi$-fuzzy contractive mappings as follows:

Definition 11 ([23]). Let $(\mathcal{S}, \mathcal{L}, *)$ be a FMS and $\alpha, \eta: \mathcal{S} \times \mathcal{S} \times(0, \infty) \rightarrow[0, \infty)$ be two functions. We say that $\mathcal{G}: \mathcal{S} \rightarrow \mathcal{S}$ is a modified $\alpha$ - $\phi$-fuzzy contractive mapping if there exists a function $\phi \in \Phi$ such that for all $\theta, \vartheta \in \mathcal{S}$ and all $\varsigma>0$, we have

$$
\alpha(\theta, \vartheta, \varsigma) \geq \eta(\theta, \vartheta, \varsigma) \Rightarrow\left(\frac{1}{\mathcal{L}(\mathcal{G} \theta, \mathcal{G} \vartheta, \varsigma)}-1\right) \leq \phi\left(\frac{1}{\mathcal{N}(\theta, \vartheta, \varsigma)}-1\right)
$$

where $\mathcal{N}(\theta, \vartheta, \varsigma)=\min \{\mathcal{L}(\theta, \vartheta, \varsigma), \mathcal{L}(\theta, \mathcal{G} \theta, \varsigma), \mathcal{L}(\vartheta, \mathcal{G} \vartheta, \varsigma)\}$

Here we mention that $\Phi$ is the set of right continuous functions $\phi:[0,+\infty) \rightarrow[0,+\infty)$ such that $\phi(\hbar)<\hbar$ for all $\hbar>0$.

Remark 2. If $\eta(\theta, \vartheta, \varsigma)=1$ and $\mathcal{N}(\theta, \vartheta, \varsigma)=\mathcal{L}(\theta, \vartheta, \varsigma)$, then this definition reduces to the concept of $\alpha$ - $\phi$-fuzzy contractive mapping given by Gopal et al. [25].

\section{Main Results}

Definition 12. Let $(\mathcal{S}, \mathcal{L}, *)$ be a FMS and let $\alpha, \eta: \mathcal{S} \times \mathcal{S} \times(0,+\infty) \rightarrow[0,+\infty)$ be two given functions. A mapping $\mathcal{G}: \mathcal{S} \rightarrow \mathcal{S}$ is called a generalized $\alpha-\eta-\mathcal{F} \mathcal{Z}$-contraction with respect to $\zeta \in \mathcal{F} \mathcal{Z}$ if for all $\theta, \vartheta \in \mathcal{S}, \varsigma>0$, we have

$$
\alpha(\theta, \vartheta, \varsigma) \geq \eta(\theta, \vartheta, \varsigma) \Rightarrow \zeta(\mathcal{L}(\mathcal{G} \theta, \mathcal{G} \vartheta, \varsigma), \mathcal{N}(\theta, \vartheta, \varsigma)) \geq 0
$$

where $\mathcal{N}(\theta, \vartheta, \varsigma)=\min \{\mathcal{L}(\theta, \vartheta, \varsigma), \mathcal{L}(\theta, \mathcal{G} \theta, \varsigma), \mathcal{L}(\vartheta, \mathcal{G} \vartheta, \varsigma)\}$

Remark 3. (i) If $\alpha(\theta, \vartheta, \varsigma)=\eta(\theta, \vartheta, \varsigma)=1$ for all $\theta, \vartheta \in \mathcal{S}, \varsigma>0$ and $\mathcal{N}(\theta, \vartheta, \varsigma)=\mathcal{L}(\theta, \vartheta, \varsigma)$, then Definition 12 yields the concept of $\mathcal{F} \mathcal{Z}$-contraction ([8,19]), even the definition of the fuzzy contractive mapping introduced in [5] by taking $\zeta(\omega, \omega)=\kappa\left(\frac{1}{\omega}-1\right)-\frac{1}{\omega}+1, \kappa \in(0,1)$.

(ii) If $\phi \in \Phi$ and define $\zeta(\omega, \omega)=\phi\left(\frac{1}{\omega}-1\right)-\frac{1}{\omega}+1$ for all $\omega, \omega \in(0,1]$, then this definition yields the definition of modified $\alpha$ - $\phi$-fuzzy contractive mapping introduced by Mishra, Vetro, and Kumam in [23].

Definition 13. Let $(\mathcal{S}, \mathcal{L}, *)$ be a FMS and let $\alpha, \eta: \mathcal{S} \times \mathcal{S} \times(0,+\infty) \rightarrow[0,+\infty)$ be two given functions. A mapping $\mathcal{G}: \mathcal{S} \rightarrow \mathcal{S}$ is called a modified $\alpha-\eta$ - $\mathcal{F} \mathcal{Z}$-contraction with respect to $\zeta \in \mathcal{F} \mathcal{Z}$ if for all $\theta, \vartheta \in \mathcal{S}, \varsigma>0$, we have

$$
\begin{aligned}
\alpha(\theta, \mathcal{G} \theta, \varsigma) \alpha(\vartheta, \mathcal{G} \vartheta, \varsigma) \geq \eta(\theta, \mathcal{G} \theta, \varsigma) \eta(\vartheta, \mathcal{G} \vartheta, \varsigma) \\
\\
\Rightarrow \zeta(\mathcal{L}(\mathcal{G} \theta, \mathcal{G} \vartheta, \varsigma), \quad \mathcal{K}(\theta, \vartheta, \varsigma)) \geq 0
\end{aligned}
$$

where $\mathcal{K}(\theta, \vartheta, \varsigma)=\min \{\mathcal{L}(\theta, \vartheta, \varsigma), \max \{\mathcal{L}(\theta, \mathcal{G} \theta, \varsigma), \mathcal{L}(\vartheta, \mathcal{G} \vartheta, \varsigma)\}\}$. 
Remark 4. If $\psi \in \Psi$ and we define $\zeta:(0,1] \times(0,1] \longrightarrow \mathbb{R}$ by $\zeta(\omega, \omega)=\frac{1}{\psi(\omega)}-\frac{1}{\omega}$ for all $\omega, \omega \in(0,1]$, then this definition leads to the notion of modified $\alpha-\eta-\psi-f u z z y$ contractive mapping stated as Definition 4.1 in [12].

Now, we give our first main result.

Theorem 1. Let $(\mathcal{S}, \mathcal{L}, *)$ be a complete FMS and let $\alpha, \eta: \mathcal{S} \times \mathcal{S} \times(0,+\infty) \rightarrow[0,+\infty)$ be two given functions and $\mathcal{G}: \mathcal{S} \longrightarrow \mathcal{S}$ be a generalized $\alpha-\eta-\mathcal{F} \mathcal{Z}$-contraction with respect to $\zeta \in \mathcal{F} \mathcal{Z}$ such that

(i) $\mathcal{G}$ is triangular $\alpha$-orbital admissible with respect to $\eta$;

(ii) there exists $\theta_{0} \in \mathcal{S}$ such that $\alpha\left(\theta_{0}, \mathcal{G} \theta_{0}, \varsigma\right) \geq \eta\left(\theta_{0}, \mathcal{G} \theta_{0}, \varsigma\right)$;

(iii) $\mathcal{G}$ is continuous.

Then, there exists $\theta^{*} \in \mathcal{S}$ such that $\mathcal{G} \theta^{*}=\theta^{*}$.

Proof. Using condition (ii), there exists $\theta_{0} \in \mathcal{S}$ with $\alpha\left(\theta_{0}, \mathcal{G} \theta_{0}, \varsigma\right) \geq \eta\left(\theta_{0}, \mathcal{G} \theta_{0}, \varsigma\right)$. Define a sequence $\left\{\theta_{n}\right\}$ in $\mathcal{S}$ by

$$
\theta_{n+1}=\mathcal{G} \theta_{n}, \text { for all } n \in \mathbb{N} \text {. }
$$

If $\theta_{m+1}=\mathcal{G} \theta_{m}$ for some $m \in \mathbb{N}$, then $\mathcal{G}$ has a fixed point. Thus, we suppose that $\theta_{n+1} \neq \theta_{n}$ for all $n \in \mathbb{N}$. As $\mathcal{G}$ is an $\alpha$-orbital admissible mapping with respect to $\eta$, we have

$$
\begin{aligned}
& \alpha\left(\theta_{0}, \theta_{1}, \varsigma\right)=\alpha\left(\theta_{0}, \mathcal{G} \theta_{0}, \varsigma\right) \geq \eta\left(\theta_{0}, \mathcal{G} \theta_{0}, \varsigma\right)=\eta\left(\theta_{0}, \theta_{1}, \varsigma\right) \text { implies that } \\
& \alpha\left(\theta_{1}, \theta_{2}, \varsigma\right)=\alpha\left(\mathcal{G} \theta_{0}, \mathcal{G} \theta_{1}, \varsigma\right) \geq \eta\left(\mathcal{G} \theta_{0}, \mathcal{G} \theta_{1}, \varsigma\right)=\eta\left(\theta_{1}, \theta_{2}, \varsigma\right) .
\end{aligned}
$$

Recursively, we get that

$$
\alpha\left(\theta_{n}, \theta_{n+1}, \varsigma\right) \geq \eta\left(\theta_{n}, \theta_{n+1}, \varsigma\right), \text { for all } n \in \mathbb{N} .
$$

Keeping in mind that $\mathcal{G}$ is a generalized $\alpha-\eta-\mathcal{F} \mathcal{Z}$-contraction and taking $\theta=\theta_{n}$ and $\vartheta=\theta_{n-1}$ in (2), we get

$$
\begin{aligned}
0 & \leq \zeta\left(\mathcal{L}\left(\mathcal{G} \theta_{n}, \mathcal{G} \theta_{n-1}, \varsigma\right), \mathcal{N}\left(\theta_{n}, \theta_{n-1}, \varsigma\right)\right) \\
& =\zeta\left(\mathcal{L}\left(\theta_{n+1}, \theta_{n}, \varsigma\right), \mathcal{N}\left(\theta_{n}, \theta_{n-1}, \varsigma\right)\right) \\
& <\frac{1}{\mathcal{N}\left(\theta_{n}, \theta_{n-1}, \varsigma\right)}-\frac{1}{\mathcal{L}\left(\theta_{n+1}, \theta_{n}, \varsigma\right)}
\end{aligned}
$$

Hence

$$
\frac{1}{\mathcal{L}\left(\theta_{n+1}, \theta_{n}, \varsigma\right)}<\frac{1}{\mathcal{N}\left(\theta_{n}, \theta_{n-1}, \varsigma\right)}
$$

which is equivalent to

$$
\mathcal{N}\left(\theta_{n}, \theta_{n-1}, \varsigma\right)<\mathcal{L}\left(\theta_{n+1}, \theta_{n}, \varsigma\right)
$$

where

$$
\begin{aligned}
\mathcal{N}\left(\theta_{n}, \theta_{n-1}, \varsigma\right) & =\min \left\{\mathcal{L}\left(\theta_{n}, \theta_{n-1}, \varsigma\right), \mathcal{L}\left(\theta_{n}, \mathcal{G} \theta_{n}, \varsigma\right), \mathcal{L}\left(\theta_{n-1}, \mathcal{G} \theta_{n-1}, \varsigma\right)\right\} \\
& =\min \left\{\mathcal{L}\left(\theta_{n}, \theta_{n-1}, \varsigma\right), \mathcal{L}\left(\theta_{n}, \theta_{n+1}, \varsigma\right), \mathcal{L}\left(\theta_{n-1}, \theta_{n}, \varsigma\right)\right\} \\
& =\min \left\{\mathcal{L}\left(\theta_{n}, \theta_{n+1}, \varsigma\right), \mathcal{L}\left(\theta_{n-1}, \theta_{n}, \varsigma\right)\right\} .
\end{aligned}
$$

Now if $\min \left\{\mathcal{L}\left(\theta_{n}, \theta_{n+1}, \varsigma\right), \mathcal{L}\left(\theta_{n-1}, \theta_{n}, \varsigma\right)\right\}=\mathcal{L}\left(\theta_{n}, \theta_{n+1}, \varsigma\right)$, then

$$
\mathcal{L}\left(\theta_{n}, \theta_{n+1}, \varsigma\right)<\mathcal{L}\left(\theta_{n+1}, \theta_{n}, \varsigma\right)
$$


which leads to a contradiction. Then

$$
\min \left\{\mathcal{L}\left(\theta_{n}, \theta_{n+1}, \varsigma\right), \mathcal{L}\left(\theta_{n-1}, \theta_{n}, \varsigma\right)\right\}=\mathcal{L}\left(\theta_{n-1}, \theta_{n}, \varsigma\right)
$$

Moreover, by (5), we obtain that

$$
\mathcal{L}\left(\theta_{n-1}, \theta_{n}, \varsigma\right)<\mathcal{L}\left(\theta_{n}, \theta_{n+1}, \varsigma\right) \text { for all } n \in \mathbb{N}
$$

As a result, $\left\{\mathcal{L}\left(\theta_{n}, \theta_{n+1}, \varsigma\right)\right\}$ is a nondecreasing sequence of positive real numbers in $[0,1]$. Thus, there exists $l(\varsigma) \leq 1$ such that $\lim _{n \rightarrow \infty} \mathcal{L}\left(\theta_{n}, \theta_{n-1}, \varsigma\right)=l(\varsigma) \leq 1$ for all $\varsigma>0$. We claim that

$$
\lim _{n \rightarrow+\infty} \mathcal{L}\left(\theta_{n}, \theta_{n-1}, \varsigma\right)=1
$$

Assume on the contrary that $l\left(\varsigma_{0}\right)<1$ for some $\varsigma_{0}>0$. Now, taking $\tau_{n}=\mathcal{L}\left(\theta_{n}, \theta_{n+1}, \varsigma_{0}\right)$ and $\delta_{n}=\mathcal{L}\left(\theta_{n-1}, \theta_{n}, \varsigma_{0}\right)$, together with condition $(\zeta 3)$, we obtain that

$$
0 \leq \lim _{n \rightarrow+\infty} \sup \zeta\left(\mathcal{L}\left(\theta_{n}, \theta_{n+1}, \varsigma_{0}\right), \mathcal{L}\left(\theta_{n-1}, \theta_{n}, \varsigma_{0}\right)\right)<0,
$$

which leads a contraction and yields $\lim _{n \rightarrow+\infty} \mathcal{L}\left(\theta_{n}, \theta_{n+1}, \varsigma\right)=1$ for all $\varsigma>0$.

Next, we reason by contradiction to show that $\left\{\theta_{n}\right\}$ is a Cauchy sequence in $\mathcal{S}$. Assume that $\left\{\theta_{n}\right\}$ is not a Cauchy sequence. Thus, there exist $\epsilon \in(0,1), \varsigma_{0}>0$ and two subsequences $\left\{\theta_{n_{k}}\right\}$ and $\left\{\theta_{m_{k}}\right\}$ of $\left\{\theta_{n}\right\}$ with $m_{k}>n_{k} \geq k$ for all $k \in \mathbb{N}$ such that

$$
\mathcal{L}\left(\theta_{n_{k}}, \theta_{m_{k}}, \varsigma_{0}\right) \leq 1-\epsilon .
$$

By Lemma 1, we derive that

$$
\mathcal{L}\left(\theta_{n_{k}}, \theta_{m_{k}}, \frac{\varsigma_{0}}{2}\right) \leq 1-\epsilon
$$

By choosing $m_{k}$ as the smallest index satisfying (9), we obtain

$$
\mathcal{L}\left(\theta_{n_{k}}, \theta_{m_{k}-1}, \frac{\varsigma_{0}}{2}\right)>1-\epsilon .
$$

On account of (8) and (10), and the triangular inequality, we obtain

$$
\begin{aligned}
1-\epsilon & \geq \mathcal{L}\left(\theta_{n_{k}}, \theta_{m_{k}}, \varsigma_{0}\right) \\
& \geq \mathcal{L}\left(\theta_{n_{k}}, \theta_{m_{k}-1}, \frac{\varsigma_{0}}{2}\right) * \mathcal{L}\left(\theta_{m_{k}-1}, \theta_{m_{k}}, \frac{\varsigma_{0}}{2}\right) \\
& >(1-\epsilon) * \mathcal{L}\left(\theta_{m_{k}-1}, \theta_{m_{k}}, \frac{\varsigma_{0}}{2}\right) .
\end{aligned}
$$

Taking the limits in the above inequality as $k \rightarrow+\infty$, making use of (7), we conclude that

$$
\lim _{k \rightarrow+\infty} \mathcal{L}\left(\theta_{n_{k}}, \theta_{m_{k}}, \varsigma_{0}\right)=1-\epsilon
$$

Furthermore, since $\mathcal{G}$ is triangular $\alpha$-orbital admissible with respect to $\eta$, we obtain

$$
\alpha\left(\theta_{n_{k}-1}, \theta_{m_{k}-1}, \varsigma_{0}\right) \geq \eta\left(\theta_{n_{k}-1}, \theta_{m_{k}-1}, \varsigma_{0}\right) .
$$


Since $\mathcal{G}$ is a generalized $\alpha-\eta-\mathcal{F} \mathcal{Z}$-contraction with respect to $\zeta \in \mathcal{F} \mathcal{Z}$, we obtain that

$$
\begin{aligned}
0 & \leq \zeta\left(\mathcal{L}\left(\mathcal{G} \theta_{n_{k}-1}, \mathcal{G} \theta_{m_{k}-1}, \varsigma_{0}\right), \mathcal{N}\left(\theta_{n_{k}-1}, \theta_{m_{k}-1}, \varsigma_{0}\right)\right) \\
& =\zeta\left(\mathcal{L}\left(\theta_{n_{k}}, \theta_{m_{k}}, \varsigma_{0}\right), \mathcal{N}\left(\theta_{n_{k}-1}, \theta_{m_{k}-1}, \varsigma_{0}\right)\right) \\
& <\frac{1}{\mathcal{N}\left(\theta_{n_{k}-1}, \theta_{m_{k}-1}, \varsigma_{0}\right)}-\frac{1}{\mathcal{L}\left(\theta_{n_{k}}, \theta_{m_{k}}, \varsigma_{0}\right)}
\end{aligned}
$$

which implies that

$$
\mathcal{N}\left(\theta_{n_{k}-1}, \theta_{m_{k}-1}, \varsigma_{0}\right) \quad<\mathcal{L}\left(\theta_{n_{k}}, \theta_{m_{k}}, \varsigma_{0}\right)
$$

where

$$
\begin{aligned}
\mathcal{N}\left(\theta_{n_{k}-1}, \theta_{m_{k}-1}, \varsigma_{0}\right) & =\min \left\{\mathcal{L}\left(\theta_{n_{k}-1}, \theta_{m_{k}-1}, \varsigma_{0}\right), \mathcal{L}\left(\theta_{n_{k}-1}, \mathcal{G} \theta_{n_{k}-1}, \varsigma_{0}\right), \mathcal{L}\left(\theta_{m_{k}-1}, \mathcal{G} \theta_{m_{k}-1}, \varsigma_{0}\right)\right\} \\
& =\min \left\{\mathcal{L}\left(\theta_{n_{k}-1}, \theta_{m_{k}-1}, \varsigma_{0}\right), \mathcal{L}\left(\theta_{n_{k}-1}, \theta_{n_{k}}, \varsigma_{0}\right), \mathcal{L}\left(\theta_{m_{k}-1}, \theta_{m_{k}}, \varsigma_{0}\right)\right\} .
\end{aligned}
$$

Now, if $\mathcal{N}\left(\theta_{n_{k}-1}, \theta_{m_{k}-1}, \varsigma_{0}\right)=\mathcal{L}\left(\theta_{m_{k}-1}, \theta_{m_{k}}, \varsigma_{0}\right)$, then it follows from (13) that

$$
\mathcal{L}\left(\theta_{m_{k}-1}, \theta_{m_{k}}, \varsigma_{0}\right)<\mathcal{L}\left(\theta_{n_{k}}, \theta_{m_{k}}, \varsigma_{0}\right)
$$

Taking the limits as $k \rightarrow \infty$ and employing (7), we obtain

$$
1 \leq \lim _{k \rightarrow+\infty} \mathcal{L}\left(x_{n_{k}}, x_{m_{k}}, \varsigma_{0}\right)
$$

consequently $\lim _{k \rightarrow+\infty} \mathcal{L}\left(x_{n_{k}}, x_{m_{k}}, \varsigma_{0}\right)=1$, which contradicts (8). Applying the same reasoning to the case $\mathcal{N}\left(\theta_{n_{k}-1}, \theta_{m_{k}-1}, \varsigma_{0}\right)=\mathcal{L}\left(\theta_{n_{k}-1}, \theta_{n_{k}}, \varsigma_{0}\right)$, we have the same conclusion. As a result, we must have $\mathcal{N}\left(\theta_{n_{k}-1}, \theta_{m_{k}-1}, \varsigma_{0}\right)=\mathcal{L}\left(\theta_{n_{k}-1}, \theta_{m_{k}-1}, \varsigma_{0}\right)$ and by (13) we derive that

$$
\mathcal{L}\left(\theta_{n_{k}-1}, \theta_{m_{k}-1}, \varsigma_{0}\right) \quad<\mathcal{L}\left(\theta_{n_{k}}, \theta_{m_{k}}, \varsigma_{0}\right) .
$$

From (8), (10), and (14), it follows that

$$
\begin{aligned}
1-\epsilon & \geq \mathcal{L}\left(\theta_{n_{k}}, \theta_{m_{k}}, \varsigma_{0}\right) \\
& >\mathcal{L}\left(\theta_{n_{k}-1}, \theta_{m_{k}}-1, \varsigma_{0}\right) \\
& \geq \mathcal{L}\left(\theta_{n_{k}-1}, \theta_{n_{k}}, \frac{\varsigma_{0}}{2}\right) * \mathcal{L}\left(\theta_{n_{k}}, \theta_{m_{k}-1}, \frac{\varsigma_{0}}{2}\right) \\
& >\mathcal{L}\left(\theta_{n_{k}-1}, \theta_{n_{k}}, \frac{\varsigma_{0}}{2}\right) *(1-\epsilon) .
\end{aligned}
$$

Letting $k \rightarrow \infty$ in the above inequality and applying (7), we get

$$
\lim _{k \rightarrow \infty} \mathcal{L}\left(\theta_{n_{k}-1}, \theta_{m_{k}-1}, \varsigma_{0}\right)=1-\epsilon=\lim _{k \rightarrow \infty} \mathcal{L}\left(\theta_{n_{k}}, \theta_{m_{k}}, \varsigma_{0}\right) .
$$

Taking the sequences $\omega_{k}=\mathcal{L}\left(\theta_{n_{k}-1}, \theta_{m_{k}-1}, \varsigma_{0}\right)$ and $\omega_{k}=\mathcal{L}\left(\theta_{m_{k}}, \theta_{n_{k}}, \varsigma_{0}\right)$ and applying (Ъ3), we deduce that

$$
0 \leq \lim _{k \rightarrow \infty} \sup \zeta\left(\mathcal{L}\left(\theta_{m_{k}}, \theta_{n_{k}}, \varsigma_{0}\right), \mathcal{L}\left(\theta_{n_{k}-1}, \theta_{m_{k}-1}, \varsigma_{0}\right)\right)<0,
$$

a contradiction. Hence, $\left\{\theta_{n}\right\}$ is a Cauchy sequence. As $(\mathcal{S}, \mathcal{L}, *)$ is a complete FMS, there exists $\theta^{*} \in \mathcal{S}$ such that

$$
\lim _{n \rightarrow \infty} \mathcal{L}\left(\theta_{n}, \theta^{*}, \varsigma\right)=1
$$


As $\mathcal{G}$ is continuous, we conclude that

$$
\lim _{n \rightarrow \infty} \mathcal{L}\left(\theta_{n+1}, \mathcal{G} \theta^{*}, \varsigma\right)=\lim _{n \rightarrow \infty} \mathcal{L}\left(\mathcal{G} \theta_{n}, \mathcal{G} \theta^{*}, \varsigma\right)=1
$$

We deduce from the uniqueness of the limit that $\theta^{*}$ is fixed point of $\mathcal{G}$, that is that $\mathcal{G} \theta^{*}=\theta^{*}$.

Theorem 2. Let $(\mathcal{S}, \mathcal{L}, *)$ be a complete FMS and let $\alpha, \eta: \mathcal{S} \times \mathcal{S} \times(0,+\infty) \rightarrow[0,+\infty)$ be two given functions and $\mathcal{G}: \mathcal{S} \longrightarrow \mathcal{S}$ be a generalized $\alpha-\eta-\mathcal{F} \mathcal{Z}$-contraction with respect to $\zeta \in \mathcal{F} \mathcal{Z}$ satisfying the following conditions:

(i) $\mathcal{G}$ is triangular $\alpha$-orbital admissible with respect to $\eta$;

(ii) there exists $\theta_{0} \in \mathcal{S}$ such that $\alpha\left(\theta_{0}, \mathcal{G} \theta_{0}, \varsigma\right) \geq \eta\left(\theta_{0}, \mathcal{G} \theta_{0}, \varsigma\right)$;

(iii) if $\left\{\theta_{n}\right\}$ is a sequence in $\mathcal{S}$ such that $\alpha\left(\theta_{n}, \theta_{n+1}, \varsigma\right) \geq \eta\left(\theta_{n}, \theta_{n+1}, \varsigma\right)$ for all $n \in \mathbb{N}, \varsigma>0$ and $\theta_{n} \rightarrow \theta \in \mathcal{S}$, then there exists a subsequence $\left\{\theta_{n(k)}\right\}$ of $\left\{\theta_{n}\right\}$ such that $\alpha\left(\theta_{n(k)}, \theta, \varsigma\right) \geq$ $\eta\left(\theta_{n(k)}, \theta, \varsigma\right)$ for all $k \in \mathbb{N}$ and $\varsigma>0$.

Then, there exists $\theta^{*} \in \mathcal{S}$ such that $\mathcal{G} \theta^{*}=\theta^{*}$.

Proof. Regarding the proof of Theorem 1, we obtain that the sequence $\left\{\theta_{n}\right\}$ given by $\theta_{n+1}=\mathcal{G} \theta_{n}$ for all $n \geq 0$ is a Cauchy in $\mathcal{S}$. As $(\mathcal{S}, \mathcal{L}, *)$ is a complete FMS, there exists $\theta^{*} \in \mathcal{S}$ such that $\theta_{n} \rightarrow \theta^{*}$ as $n \rightarrow \infty$. By the condition (iii), there exists a subsequence $\left\{\theta_{n(k)}\right\}$ of $\left\{\theta_{n}\right\}$ such that $\alpha\left(\theta_{n(k)}, \theta^{*}, \varsigma\right) \geq \eta\left(\theta_{n(k)}, \theta^{*}, \varsigma\right)$ for all $k \in \mathbb{N}, \varsigma>0$. Applying (2), we have

$$
\begin{aligned}
0 & \leq \zeta\left(\mathcal{L}\left(\mathcal{G} \theta_{n(k)}, \mathcal{G} \theta^{*}, \varsigma\right), \mathcal{N}\left(\theta_{n(k)}, \theta^{*}, \varsigma\right)\right) \\
& =\zeta\left(\mathcal{L}\left(\theta_{n(k)+1}, \mathcal{G} \theta^{*}, \varsigma\right), \mathcal{N}\left(\theta_{n(k)}, \theta^{*}, \varsigma\right)\right)
\end{aligned}
$$

where

$$
\begin{aligned}
\mathcal{N}\left(\theta_{n(k)}, \theta^{*}, \varsigma\right) & =\min \left\{\mathcal{L}\left(\theta_{n(k)}, \theta^{*}, \varsigma\right), \mathcal{L}\left(\theta_{n(k)}, \mathcal{G} \theta_{n(k)}, \varsigma\right), L\left(\theta^{*}, \mathcal{G} \theta^{*}, \varsigma\right)\right\} \\
& =\min \left\{\mathcal{L}\left(\theta_{n(k)}, \theta^{*}, \varsigma\right), \mathcal{L}\left(\theta_{n(k)}, \theta_{n(k)+1}, \varsigma\right), \mathcal{L}\left(\theta^{*}, \mathcal{G} \theta^{*}, \varsigma\right)\right\}
\end{aligned}
$$

Taking $k \rightarrow \infty$ in $\mathcal{N}\left(\theta_{n(k)}, \theta^{*}, \varsigma\right)$, we get

$$
\begin{aligned}
\lim _{k \rightarrow \infty} \mathcal{N}\left(\theta_{n(k)}, \theta^{*}, \varsigma\right) & =\min \left\{1,1, \mathcal{L}\left(\theta^{*}, \mathcal{G} \theta^{*}, \varsigma\right)\right\} \\
& =\mathcal{L}\left(\theta^{*}, \mathcal{G} \theta^{*}, \varsigma\right) .
\end{aligned}
$$

Suppose that $\mathcal{G} \theta^{*} \neq \theta^{*}$. Then $\mathcal{L}\left(\theta^{*}, \mathcal{G} \theta^{*}, \varsigma\right)<1$. Now by choosing the sequences $\tau_{k}=\mathcal{L}\left(\theta_{n(k)+1}, \mathcal{G} \theta^{*}, \varsigma\right)$ and $\sigma_{k}=N\left(\theta_{n(k)}, \theta^{*}, \varsigma\right)$, we have $\lim _{k \rightarrow+\infty} \tau_{k}=\lim _{k \rightarrow+\infty} \sigma_{k}<1$.

Applying the condition $(\zeta 3)$, it follows that

$$
0 \leq \lim _{k \rightarrow \infty} \sup \zeta\left(\mathcal{L}\left(\theta_{n(k)+1}, \mathcal{G} \theta^{*}, \varsigma\right), \mathcal{N}\left(\theta_{n(k)}, \theta^{*}, \varsigma\right)\right)<0,
$$

which is a contradiction. Then $\mathcal{L}\left(\theta^{*}, \mathcal{G} \theta^{*}, \varsigma\right)=1$, that is $\mathcal{G} \theta^{*}=\theta^{*}$.

Example 3. Let $\mathcal{S}=[0, \infty)$ equipped with the fuzzy metric $\mathcal{L}: \mathcal{S} \times \mathcal{S} \times(0, \infty) \longrightarrow[0,1)$ defined by $\mathcal{L}(\theta, \vartheta, \varsigma)=\frac{\varsigma}{\varsigma+|\theta-\vartheta|}$ for all $\theta, \vartheta \in \mathcal{S}, \varsigma>0$ and $*$ is the product $t$-norm. We define $\mathcal{G}: \mathcal{S} \longrightarrow \mathcal{S}$ by

$$
\mathcal{G} \theta=\left\{\begin{array}{cc}
\frac{\theta^{2}}{6} & \text { if } \theta \in[0,1], \\
\frac{3}{2} & \text { otherwise, }
\end{array}\right.
$$


and $\alpha, \eta: \mathcal{S} \times \mathcal{S} \times(0, \infty) \longrightarrow[0, \infty)$ by

$$
\begin{aligned}
& \alpha(\theta, \vartheta, \varsigma)=\left\{\begin{array}{cc}
2+\theta \vartheta & \text { if } \theta, \vartheta \in[0,1], \\
0 & \text { otherwise, }
\end{array}\right. \\
& \eta(\theta, \vartheta, \varsigma)=\left\{\begin{array}{cc}
1+\theta \vartheta & \text { if } \theta, \vartheta \in[0,1], \\
6 & \text { otherwise, }
\end{array}\right.
\end{aligned}
$$

Let $(\theta, \vartheta) \in \mathcal{S} \times \mathcal{S}$, from the definition of $\alpha$ and $\eta$, we have that $\alpha(\theta, \vartheta, \varsigma) \geq \eta(\theta, \vartheta, \varsigma)$ for all $\varsigma>0$ if and only if $\theta, \vartheta \in[0,1]$. Suppose that $\alpha(\theta, \mathcal{G} \theta, \varsigma) \geq \eta(\theta, \mathcal{G} \theta, \varsigma)$ therefore $\theta, \mathcal{G} \theta \in[0,1]$, which implies that $\alpha\left(\mathcal{G} \theta, \mathcal{G}^{2} \theta, \varsigma\right) \geq \eta\left(\mathcal{G} \theta, \mathcal{G}^{2} \theta, \varsigma\right)$. Thus, $\mathcal{G}$ is an $\alpha$-orbital admissible with respect to $\eta$.

Suppose that $\alpha(\theta, \vartheta, \varsigma) \geq \eta(\theta, \vartheta, \varsigma)$ and $\alpha(\vartheta, \mathcal{G} \vartheta, \varsigma) \geq \eta(\vartheta, \mathcal{G} \vartheta, \varsigma)$ for all $\varsigma>0$, then $\theta, \vartheta, \mathcal{G} \vartheta \in[0,1]$, which implies that $\alpha(\theta, \mathcal{G} \vartheta, \varsigma) \geq \eta(\theta, \mathcal{G} \vartheta, \varsigma)$. Hence $\mathcal{G}$ is a triangular $\alpha$-orbital admissible with respect to $\eta$.

Clearly, for any $\theta_{0} \in[0,1]$ we have $\alpha\left(\theta_{0}, \mathcal{G} \theta_{0}, \varsigma\right) \geq \eta\left(\theta_{0}, \mathcal{G} \theta_{0}, \varsigma\right)$ for all $\varsigma>0$.

Next, let $\left\{\theta_{n}\right\}$ be a sequence such that $\alpha\left(\theta_{n}, \theta_{n+1}, \varsigma\right) \geq \eta\left(\theta_{n}, \theta_{n+1}, \varsigma\right)$ for all $n \in \mathbb{N}$ and $\theta_{n} \rightarrow \theta \in \mathcal{S}$ as $n \rightarrow \infty$. Hence $\left\{\theta_{n}\right\} \subseteq[0,1]$ and then $\theta \in[0,1]$, which implies that $\alpha\left(\theta_{n}, \theta, \varsigma\right) \geq$ $\eta\left(\theta_{n}, \theta, \varsigma\right)$.

Now, we have to show that (2) is satisfied. Define $\zeta:(0,1] \times(0,1] \longrightarrow \mathbb{R}$ by $\zeta(\omega, \omega)=$ $\frac{1}{3}\left(\frac{1}{\omega}-1\right)-\frac{1}{\omega}+1$, and $(\theta, \vartheta) \in \mathcal{S} \times \mathcal{S}$ with $\alpha(\theta, \vartheta, \varsigma) \geq \eta(\theta, \vartheta, \varsigma)$ for all $\varsigma>0$, that is, $\theta, \vartheta \in[0,1]$, then we have

$$
\begin{aligned}
\frac{1}{\mathcal{L}(\mathcal{G} \theta, \mathcal{G} \vartheta, \varsigma)}-1 & =\frac{\varsigma+|\mathcal{G} \theta-\mathcal{G} \vartheta|}{\varsigma}-1 \\
& =\frac{\left|\theta^{2}-\vartheta^{2}\right|}{6 \varsigma} \\
& \leq \frac{|\theta-\vartheta|}{3 \zeta} \\
& \leq \frac{1}{3 \zeta} \max \{|\theta-\vartheta|,|\theta-\mathcal{G} \theta|,|\vartheta-\mathcal{G} \vartheta|\} \\
& =\frac{1}{3}\left(\frac{1}{\mathcal{N}(\theta, \vartheta, \varsigma)}-1\right) .
\end{aligned}
$$

It follows that $\zeta(\mathcal{L}(\mathcal{G} \theta, \mathcal{G} \vartheta, \varsigma), \mathcal{N}(\theta, \vartheta, \varsigma)) \geq 0$.

Thus, $\mathcal{G}$ satisfies all the hypothesis of Theorem 2 and $\theta=0, \theta=\frac{3}{2}$ are fixed points of $\mathcal{G}$.

Note that, $\mathcal{G}$ is not a fuzzy contractive mapping [5]. By choosing $\theta=1$ and $\vartheta=\frac{3}{2}$, there is no $\kappa \in(0,1)$ satisfying

$$
\begin{aligned}
\frac{1}{\mathcal{L}(\mathcal{G} \theta, \mathcal{G} \vartheta, \varsigma)}-1=\frac{|\mathcal{G} \theta-\mathcal{G} \vartheta|}{\varsigma} & =\frac{8}{6 \varsigma} \\
& \leq \frac{\kappa}{2 \varsigma}=\kappa\left(\frac{1}{\mathcal{L}(\theta, \vartheta, \varsigma)}-1\right)
\end{aligned}
$$

To secure the uniqueness of the fixed point for generalized $\alpha-\eta-\mathcal{F} \mathcal{Z}$-contraction mapping with respect to $\zeta \in \mathcal{F} \mathcal{Z}$, the following criterion will be considered.

(C) For all $\theta, \vartheta \in \operatorname{Fix}(\mathcal{G})$, we have $\alpha(\theta, \vartheta, \varsigma) \geq \eta(\theta, \vartheta, \varsigma)$, where $\operatorname{Fix}(\mathcal{G})$ represents the collection of fixed points of $\mathcal{G}$.

Theorem 3. Adding hypothesis (C) to the assumptions of Theorem 1 (resp. Theorem 2), we get the uniqueness of the fixed point of $\mathcal{G}$. 
Proof. We argue by contradiction, assume that $\theta^{*}, \vartheta^{*} \in \mathcal{S}$ are two distinct fixed points. Thus, $\mathcal{L}\left(\theta^{*}, \vartheta^{*}, \varsigma\right)<1$ for all $\varsigma>0$. From assumption $(\mathbf{C})$, we have

$$
\alpha\left(\theta^{*}, \vartheta^{*}, \varsigma\right) \geq \eta\left(\theta^{*}, \vartheta^{*}, \varsigma\right)
$$

From (2) and ( 32$)$, we get

$$
\begin{aligned}
0 & \leq \zeta\left(\mathcal{L}\left(\mathcal{G} \theta^{*}, \mathcal{G} \vartheta^{*}, \varsigma\right), \mathcal{N}\left(\theta^{*}, \vartheta^{*}, \varsigma\right)\right) \\
& =\zeta\left(\mathcal{L}\left(\mathcal{G} \theta^{*}, \mathcal{G} \vartheta^{*}, \varsigma\right), \min \left\{\mathcal{L}\left(\theta^{*}, \vartheta^{*}, \varsigma\right), \mathcal{L}\left(\theta^{*}, \mathcal{G} \theta^{*}, \varsigma\right), \mathcal{L}\left(\vartheta^{*}, \mathcal{G} \vartheta^{*}, \varsigma\right)\right\}\right) \\
& =\zeta\left(\mathcal{L}\left(\theta^{*}, \vartheta^{*}, \varsigma\right), \min \left\{\mathcal{L}\left(\theta^{*}, \vartheta^{*}, \varsigma\right), 1,1\right\}\right) \\
& =\zeta\left(\mathcal{L}\left(\theta^{*}, \vartheta^{*}, \varsigma\right), \mathcal{L}\left(\theta^{*}, \vartheta^{*}, \varsigma\right)\right) \\
& <\frac{1}{\mathcal{L}\left(\theta^{*}, \vartheta^{*}, \varsigma\right)}-\frac{1}{\mathcal{L}\left(\theta^{*}, \vartheta^{*}, \varsigma\right)}=0,
\end{aligned}
$$

a contradiction. Then, the fixed point of $\mathcal{G}$ is unique.

Now, we derive several corollaries which can be inferred easily from our main results.

Corollary 1. Let $(\mathcal{S}, \mathcal{L}, *)$ be a complete FMS and $\mathcal{G}: \mathcal{S} \longrightarrow \mathcal{S}$ be a mapping such that for all $\theta, \vartheta \in \mathcal{S}, \varsigma>0$ and $\zeta \in \mathcal{F} \mathcal{Z}$

$$
\zeta(\mathcal{L}(\mathcal{G} \theta, \mathcal{G} \vartheta, \varsigma), \mathcal{N}(\theta, \vartheta, \varsigma)) \geq 0
$$

Then $\mathcal{G}$ has a unique fixed point.

Proof. The conclusion can be drawn from Theorem 3 by taking $\alpha(\theta, \vartheta, \varsigma)=\eta(\theta, \vartheta, \varsigma)=1$ for all $\theta, \vartheta \in \mathcal{S}$ and $\varsigma>0$.

Corollary 2 ([26]). Let $(\mathcal{S}, \mathcal{L}, *)$ be a complete FMS and $\mathcal{G}: \mathcal{S} \longrightarrow \mathcal{S}$ be a mapping such that for all $\theta, \vartheta \in \mathcal{S}, \zeta>0$ and for some $k \in(0,1)$

$$
\frac{1}{\mathcal{L}(\mathcal{G} \theta, \mathcal{G} \vartheta, \varsigma)}-1 \leq k\left(\frac{1}{\mathcal{N}(\theta, \vartheta, \varsigma)}-1\right)
$$

Then, $\mathcal{G}$ has a unique fixed point.

Proof. The conclusion can be drawn from Corollary 1 by taking $\zeta(\omega, \omega)=\kappa\left(\frac{1}{\omega}-1\right)-$ $\frac{1}{\omega}+1$ for all $\omega, \omega \in(0,1]$.

Corollary 3. Let $(\mathcal{S}, \mathcal{L}, *)$ be a complete FMS and let $\alpha, \eta: \mathcal{S} \times \mathcal{S} \times(0, \infty) \longrightarrow[0, \infty)$ be two functions, $\psi \in \Psi$ and $\mathcal{G}: \mathcal{S} \longrightarrow \mathcal{S}$ be a self-mapping such that for all $\theta, \vartheta \in \mathcal{S}$ and $\varsigma>0$,

$$
\alpha(\theta, \vartheta, \varsigma) \geq \eta(\theta, \vartheta, \varsigma) \Rightarrow \mathcal{L}(\mathcal{G} \theta, \mathcal{G} \vartheta, \varsigma) \geq \psi(\mathcal{N}(\theta, \vartheta, \varsigma))
$$

Suppose also that:

(i) $\mathcal{G}$ is a triangular $\alpha$-orbital admissible with respect to $\eta$;

(ii) there exists $\theta_{0} \in \mathcal{S}$ such that $\alpha\left(\theta_{0}, \mathcal{G} \theta_{0}, \varsigma\right) \geq \eta\left(\theta_{0}, \mathcal{G} \theta_{0}, \varsigma\right)$;

(iii) either $\mathcal{G}$ is continuous mapping or if $\left\{\theta_{n}\right\}$ is a sequence in $\mathcal{S}$ such that $\alpha\left(\theta_{n}, \theta_{n+1}, \varsigma\right) \geq$ $\eta\left(\theta_{n}, \theta_{n+1}, \varsigma\right)$ for all $n \in \mathbb{N}, \varsigma>0$ and $\theta_{n} \rightarrow \theta \in \mathcal{S}$ as $n \rightarrow \infty$, then there exists a subsequence $\left\{\theta_{n(k)}\right\}$ of $\left\{\theta_{n}\right\}$ such that $\alpha\left(\theta_{n(k)}, \theta, \varsigma\right) \geq \eta\left(\theta_{n(k)}, \theta, \varsigma\right)$ for all $k \in \mathbb{N}, \varsigma>0$,

(v) for all $\theta, \vartheta \in \operatorname{Fix}(\mathcal{G})$ and $\varsigma>0$, we have $\alpha(\theta, \vartheta, \varsigma) \geq \eta(\theta, \vartheta, \varsigma)$.

Then, $\mathcal{G}$ has a unique fixed point. 
Proof. Defining $\zeta \in \mathcal{F} \mathcal{Z}$ by

$$
\zeta(\omega, \omega)=\frac{1}{\psi(\omega)}-\frac{1}{\omega} \text { for all } \omega, \omega \in(0,1] .
$$

Then, the desired results follows from Theorem 3.

Corollary 4. Let $(\mathcal{S}, \mathcal{L}, *)$ be a complete FMS, $\alpha: \mathcal{S} \times \mathcal{S} \times(0, \infty) \longrightarrow[0, \infty)$ and $\mathcal{G}: \mathcal{S} \longrightarrow \mathcal{S}$. Assume that there exists $\phi \in \Phi$ such that for all $\theta, \vartheta \in \mathcal{S}, \varsigma>0$,

$$
\alpha(\theta, \vartheta, \varsigma) \geq 1 \Rightarrow\left(\frac{1}{\mathcal{L}(\mathcal{G} \theta, \mathcal{G} \vartheta, \varsigma)}-1\right) \leq \phi\left(\frac{1}{\mathcal{N}(\theta, \vartheta, \varsigma)}-1\right)
$$

where $\mathcal{N}(\theta, \vartheta, \varsigma)=\min \{\mathcal{L}(\theta, \vartheta, \varsigma), \mathcal{L}(\theta, \mathcal{G} \theta, \varsigma), \mathcal{L}(\vartheta, \mathcal{G} \vartheta, \varsigma)\}$, and suppose that:

(i) $\mathcal{G}$ is a triangular $\alpha$-orbital admissible;

(ii) there exists $\theta_{0} \in \mathcal{S}$ such that $\alpha\left(\theta_{0}, \mathcal{G} \theta_{0}, \varsigma\right) \geq 1$;

(iii) $\mathcal{G}$ is a continuous mapping or, if $\left\{\theta_{n}\right\}$ is a sequence in $\mathcal{S}$ such that $\alpha\left(\theta_{n}, \theta_{n+1}, \varsigma\right) \geq 1$ for all $n \in \mathbb{N}, \varsigma>0$ and $\theta_{n} \rightarrow \theta \in \mathcal{S}$ as $n \rightarrow \infty$, then there exists a subsequence $\left\{\theta_{n(k)}\right\}$ of $\left\{\theta_{n}\right\}$ such that $\alpha\left(\theta_{n(k)}, \theta, \varsigma\right) \geq 1$ for all $k \in \mathbb{N}, \varsigma>0$.

Then $\mathcal{G}$ has a fixed point. Furthermore, if for all $\theta, \vartheta \in$ Fix $(\mathcal{G})$ and all $\varsigma>0$, we have $\alpha(\theta, \vartheta, \varsigma) \geq 1$, then $\mathcal{G}$ has a unique fixed point.

Proof. The conclusion can be drawn from Theorem 3 by defining $\eta(\theta, \vartheta, \varsigma)=1$ for all $\theta, \vartheta \in \mathcal{S}$ and $\varsigma>0$, and $\zeta(\omega, \omega)=\phi\left(\frac{1}{\omega}-1\right)-\frac{1}{\omega}+1$ for all $\omega, \omega \in(0,1]$.

Theorem 4. Let $(\mathcal{S}, \mathcal{L}, *)$ be a complete FMS and let $\alpha, \eta: \mathcal{S} \times \mathcal{S} \times(0,+\infty) \rightarrow[0,+\infty)$ be two given functions and $\mathcal{G}: \mathcal{S} \longrightarrow \mathcal{S}$ be a modified $\alpha-\eta-\mathcal{F} \mathcal{Z}$-contraction with respect to $\zeta \in \mathcal{F} \mathcal{Z}$ satisfying the following conditions:

(i) $\mathcal{G}$ is a triangular $\alpha$-orbital admissible with respect to $\eta$;

(ii) there exists $\theta_{0} \in \mathcal{S}$ such that $\alpha\left(\theta_{0}, \mathcal{G} \theta_{0}, \varsigma\right) \geq \eta\left(\theta_{0}, \mathcal{G} \theta_{0}, \varsigma\right)$;

(iii) if $\left\{\theta_{n}\right\}$ is a sequence in $\mathcal{S}$ such that $\alpha\left(\theta_{n}, \theta_{n+1}, \varsigma\right) \geq \eta\left(\theta_{n}, \theta_{n+1}, \varsigma\right)$ for all $n \in \mathbb{N}, \varsigma>0$ and $\theta_{n} \rightarrow \theta \in \mathcal{S}$ as $n \rightarrow \infty$, then there exists a subsequence $\left\{\theta_{n(k)}\right\}$ of $\left\{\theta_{n}\right\}$ such that $\alpha\left(\theta_{n(k)}, \theta, \varsigma\right) \geq \eta\left(\theta_{n(k)}, \theta, \varsigma\right)$ and $\alpha(\theta, \mathcal{G} \theta, \varsigma) \geq \eta(\theta, \mathcal{G} \theta, \varsigma)$ for all $k \in \mathbb{N}$ and $\varsigma>0$.

Then $\mathcal{G}$ has a fixed point.

Proof. Let $\theta_{0} \in \mathcal{S}$ such that $\alpha\left(\theta_{0}, \mathcal{G} \theta_{0}, \varsigma\right) \geq \eta\left(\theta_{0}, \mathcal{G} \theta_{0}, \varsigma\right)$. Define a sequence $\left\{\theta_{n}\right\}$ in $\mathcal{S}$ by $\theta_{n+1}=\mathcal{G} \theta_{n}$ for all $n \in \mathbb{N}$. If $\theta_{m+1}=\mathcal{G} \theta_{m}$ for some $m \in \mathbb{N}$, then $\mathcal{G}$ has a fixed point. Suppose that $\theta_{n+1} \neq \theta_{n}$ for all $n \in \mathbb{N}$. As $\mathcal{G}$ is $\alpha$-admissible with respect to $\eta$ and $\alpha\left(\theta_{0}, \mathcal{G} \theta_{0}, \varsigma\right) \geq$ $\eta\left(\theta_{0}, \mathcal{G} \theta_{0}, \zeta\right)$, we have

$$
\alpha\left(\theta_{1}, \theta_{2}, \varsigma\right)=\alpha\left(\mathcal{G} \theta_{0}, \mathcal{G} \theta_{1}, \varsigma\right) \geq \eta\left(\mathcal{G} \theta_{0}, \mathcal{G} \theta_{1}, \varsigma\right)=\eta\left(\theta_{1}, \theta_{2}, \varsigma\right)
$$

By carrying forward with this process, we obtain $\alpha\left(\theta_{n}, \mathcal{G} \theta_{n}, \varsigma\right) \geq \eta\left(\theta_{n}, \mathcal{G} \theta_{n}, \varsigma\right)$. So, we obtain

$$
\alpha\left(\theta_{n-1}, \mathcal{G} \theta_{n-1}, \varsigma\right) \alpha\left(\theta_{n}, \mathcal{G} \theta_{n}, \varsigma\right) \geq \eta\left(\theta_{n-1}, \mathcal{G} \theta_{n-1}, \varsigma\right) \eta\left(\theta_{n}, \mathcal{G} \theta_{n}, \varsigma\right)
$$

for all $n \in \mathbb{N}, \varsigma>0$

As $\mathcal{G}$ is a modified $\alpha-\eta-\mathcal{F} \mathcal{Z}$-contraction, taking $\theta=\theta_{n}$ and $\vartheta=\theta_{n-1}$ in (3), we get

$$
\begin{aligned}
0 & \leq \zeta\left(\mathcal{L}\left(\mathcal{G} \theta_{n}, \mathcal{G} \theta_{n-1}, \varsigma\right), \mathcal{K}\left(\theta_{n}, \theta_{n-1}, \varsigma\right)\right) \\
& =\zeta\left(\mathcal{L}\left(\theta_{n+1}, \theta_{n}, \varsigma\right), \mathcal{K}\left(\theta_{n}, \theta_{n-1}, \varsigma\right)\right) \\
& <\frac{1}{\mathcal{K}\left(\theta_{n}, \theta_{n-1}, \varsigma\right)}-\frac{1}{\mathcal{L}\left(\theta_{n+1}, \theta_{n}, \varsigma\right)} .
\end{aligned}
$$


Hence

$$
\mathcal{K}\left(\theta_{n}, \theta_{n-1}, \varsigma\right)<\mathcal{L}\left(\theta_{n+1}, \theta_{n}, \varsigma\right)
$$

where

$$
\begin{aligned}
\mathcal{K}\left(\theta_{n}, \theta_{n-1}, \varsigma\right) & =\min \left\{\mathcal{L}\left(\theta_{n}, \theta_{n-1}, \varsigma\right), \max \left\{\mathcal{L}\left(\theta_{n}, \mathcal{G} \theta_{n}, \varsigma\right), \mathcal{L}\left(\theta_{n-1}, \mathcal{G} \theta_{n-1}, \varsigma\right)\right\}\right\} \\
& =\min \left\{\mathcal{L}\left(\theta_{n}, \theta_{n-1}, \varsigma\right), \max \left\{\mathcal{L}\left(\theta_{n}, \theta_{n+1}, \varsigma\right), \mathcal{L}\left(\theta_{n-1}, \theta_{n}, \varsigma\right)\right\}\right\}
\end{aligned}
$$
we have

If $\left.\max \left\{\mathcal{L}\left(\theta_{n}, \theta_{n+1}, \varsigma\right), \mathcal{L}\left(\theta_{n-1}, \theta_{n}, \varsigma\right)\right\}\right\}=\mathcal{L}\left(\theta_{n}, \theta_{n+1}, \varsigma\right)$ then from above inequality,

$$
\mathcal{K}\left(\theta_{n}, \theta_{n-1}, \varsigma\right)=\min \left\{\mathcal{L}\left(\theta_{n}, \theta_{n-1}, \varsigma\right), \mathcal{L}\left(\theta_{n}, \theta_{n+1}, \varsigma\right)\right\}=\mathcal{L}\left(\theta_{n}, \theta_{n-1}, \varsigma\right)
$$

On the other hand, if $\left.\max \left\{\mathcal{L}\left(\theta_{n}, \theta_{n+1}, \varsigma\right), \mathcal{L}\left(\theta_{n-1}, \theta_{n}, \varsigma\right)\right\}\right\}=\mathcal{L}\left(\theta_{n-1}, \theta_{n}, \varsigma\right)$ then, we have

$$
\mathcal{K}\left(\theta_{n}, \theta_{n-1}, \varsigma\right)=\min \left\{\mathcal{L}\left(\theta_{n}, \theta_{n-1}, \varsigma\right), \mathcal{L}\left(\theta_{n}, \theta_{n-1}, \varsigma\right)\right\}=\mathcal{L}\left(\theta_{n}, \theta_{n-1}, \varsigma\right)
$$

In both cases, we have

$$
\mathcal{K}\left(\theta_{n}, \theta_{n-1}, \varsigma\right)=\mathcal{L}\left(\theta_{n}, \theta_{n-1}, \varsigma\right)
$$

By (20), we get

$$
\mathcal{L}\left(\theta_{n-1}, \theta_{n}, \varsigma\right)<\mathcal{L}\left(\theta_{n}, \theta_{n+1}, \varsigma\right), \text { for all } n \in \mathbb{N}
$$

Then, we deduce that $\left\{\mathcal{L}\left(\theta_{n}, \theta_{n+1}, \varsigma\right)\right\}$ is a nondecreasing sequence in $[0,1]$. Thus, there exists $s(\varsigma) \leq 1$ such that $\lim _{n \rightarrow+\infty} \mathcal{L}\left(\theta_{n}, \theta_{n-1}, \varsigma\right)=s(\varsigma) \leq 1$ for all $\varsigma>0$. We claim that

$$
\lim _{n \rightarrow+\infty} \mathcal{L}\left(\theta_{n}, \theta_{n-1}, \varsigma\right)=1
$$

On the contrary, assume that $s\left(\varsigma_{0}\right)<1$ for some $\varsigma_{0}>0$. Now, consider $\tau_{n}=$ $\mathcal{L}\left(\theta_{n}, \theta_{n+1}, \varsigma_{0}\right)$ and $\delta_{n}=\mathcal{L}\left(\theta_{n-1}, \theta_{n}, \varsigma_{0}\right)$, together with condition $(\zeta 3)$, we get

$$
0 \leq \lim _{n \rightarrow \infty} \sup \zeta\left(\mathcal{L}\left(\theta_{n}, \theta_{n+1}, \varsigma_{0}\right), \mathcal{L}\left(\theta_{n-1}, \theta_{n}, \varsigma_{0}\right)\right)<0
$$

which leads to a contradiction and yields $\lim _{n \rightarrow+\infty} \mathcal{L}\left(\theta_{n}, \theta_{n+1}, \varsigma\right)=1$ for all $\varsigma>0$.

Next, we have to show that $\left\{\theta_{n}\right\}$ is a Cauchy sequence in $(\mathcal{S}, \mathcal{L}, *)$. By contradiction, assume that $\left\{\theta_{n}\right\}$ is not Cauchy. Hence, there exists $\epsilon \in(0,1), \varsigma_{0}>0$ and two subsequences $\left\{\theta_{n_{k}}\right\}$ and $\left\{\theta_{m_{k}}\right\}$ of $\left\{\theta_{n}\right\}$ where $m_{k}>n_{k} \geq k$ for all $k \in \mathbb{N}$ such that

$$
\mathcal{L}\left(\theta_{n_{k}}, \theta_{m_{k}}, \varsigma_{0}\right) \leq 1-\epsilon .
$$

By Lemma 1, we derive that

$$
\mathcal{L}\left(\theta_{n_{k}}, \theta_{m_{k}}, \frac{\varsigma_{0}}{2}\right) \leq 1-\epsilon
$$

Taking $m_{k}$ as the smallest index satisfying (23), we get

$$
\mathcal{L}\left(\theta_{n_{k}}, \theta_{m_{k}-1}, \frac{\varsigma_{0}}{2}\right)>1-\epsilon .
$$


On account of (22), (24), and (G) 4$)$, we have

$$
\begin{aligned}
1-\epsilon & \geq \mathcal{L}\left(\theta_{n_{k}}, \theta_{m_{k}}, \varsigma_{0}\right) \\
& \geq \mathcal{L}\left(\theta_{n_{k}}, \theta_{m_{k}-1}, \frac{\varsigma_{0}}{2}\right) * \mathcal{L}\left(\theta_{m_{k}-1}, \theta_{m_{k}}, \frac{\varsigma_{0}}{2}\right) \\
& >(1-\epsilon) * \mathcal{L}\left(\theta_{m_{k}-1}, \theta_{m_{k}}, \frac{\varsigma_{0}}{2}\right) .
\end{aligned}
$$

Taking the limits in the above inequality as $k \rightarrow \infty$ and using (21), we derive that

$$
\lim _{n \rightarrow \infty} \mathcal{L}\left(\theta_{n_{k}}, \theta_{m_{k}}, \varsigma_{0}\right)=1-\epsilon
$$

From the definition of modified $\alpha-\eta-\mathcal{F} \mathcal{Z}$-contraction, we have

$$
\alpha\left(\theta_{n_{k}-1}, \mathcal{G} \theta_{n_{k}-1}, \varsigma_{0}\right) \alpha\left(\theta_{m_{k}-1}, \mathcal{G} \theta_{m_{k}-1}, \varsigma_{0}\right) \geq \eta\left(\theta_{n_{k}-1}, \mathcal{G} \theta_{n_{k}-1}, \varsigma_{0}\right) \eta\left(\theta_{m_{k}-1}, \mathcal{G} \theta_{m_{k}-1}, \varsigma_{0}\right),
$$

we obtain that

$$
\begin{aligned}
0 & \leq \zeta\left(\mathcal{L}\left(\mathcal{G} \theta_{n_{k}-1}, \mathcal{G} \theta_{m_{k}-1}, \varsigma_{0}\right), \mathcal{K}\left(\theta_{n_{k}-1}, \theta_{m_{k}-1}, \varsigma_{0}\right)\right) \\
& =\zeta\left(\mathcal{L}\left(\theta_{n_{k}}, \theta_{m_{k}}, \varsigma_{0}\right), \mathcal{K}\left(\theta_{n_{k}-1}, \theta_{m_{k}-1}, \varsigma_{0}\right)\right) \\
& <\frac{1}{\mathcal{K}\left(\theta_{n_{k}-1}, \theta_{m_{k}-1}, \varsigma_{0}\right)}-\frac{1}{\mathcal{L}\left(\theta_{n_{k}}, \theta_{m_{k}}, \varsigma_{0}\right)},
\end{aligned}
$$

which implies that

$$
\mathcal{K}\left(\theta_{n_{k}-1}, \theta_{m_{k}-1}, \varsigma_{0}\right) \quad<\mathcal{L}\left(\theta_{n_{k}}, \theta_{m_{k}}, \varsigma_{0}\right)
$$

where

$$
\begin{gathered}
\mathcal{K}\left(\theta_{n_{k}-1}, \theta_{m_{k}-1}, \varsigma_{0}\right)=\min \left\{\mathcal{L}\left(\theta_{n_{k}-1}, \theta_{m_{k}-1}, \varsigma_{0}\right), \max \left\{\mathcal{L}\left(\theta_{n_{k}-1}, \mathcal{G} \theta_{n_{k}-1}, \varsigma_{0}\right), \mathcal{L}\left(\theta_{m_{k}-1}, \mathcal{G} \theta_{m_{k}-1}, \varsigma_{0}\right)\right\}\right\} \\
=\min \left\{\mathcal{L}\left(\theta_{n_{k}-1}, \theta_{m_{k}-1}, \varsigma_{0}\right), \max \left\{\mathcal{L}\left(\theta_{n_{k}-1}, \theta_{n_{k}}, \varsigma_{0}\right), \mathcal{L}\left(\theta_{m_{k}-1}, \theta_{m_{k}}, \varsigma_{0}\right)\right\}\right\} . \\
\operatorname{If~} \mathcal{K}\left(\theta_{n_{k}-1}, \theta_{m_{k}-1}, \varsigma_{0}\right)=\max \left\{\mathcal{L}\left(\theta_{n_{k}-1}, \theta_{n_{k}}, \varsigma_{0}\right), \mathcal{L}\left(\theta_{m_{k}-1}, \theta_{m_{k}}, \varsigma_{0}\right)\right\}, \text { then (25) yields } \\
\max \left\{\mathcal{L}\left(\theta_{n_{k}-1}, \theta_{n_{k}}, \varsigma_{0}\right), \mathcal{L}\left(\theta_{m_{k}-1}, \theta_{m_{k}}, \varsigma_{0}\right)\right\}<\mathcal{L}\left(\theta_{n_{k}}, \theta_{m_{k}}, \varsigma_{0}\right) .
\end{gathered}
$$

Taking the limits in the above inequality as $k \rightarrow \infty$ and using (21), we obtain

$$
\max \{1,1\}=1 \leq \lim _{k \rightarrow+\infty} \mathcal{L}\left(\theta_{n_{k}}, \theta_{m_{k}}, \varsigma_{0}\right),
$$

consequently $\lim _{k \rightarrow+\infty} \mathcal{L}\left(\theta_{n_{k}}, \theta_{m_{k}}, \varsigma_{0}\right)=1$, which contradicts (22). Therefore, we must have $\mathcal{K}\left(\theta_{n_{k}-1}, \theta_{m_{k}-1}, \varsigma_{0}\right)=\mathcal{L}\left(\theta_{n_{k}-1}, \theta_{m_{k}-1}, \varsigma_{0}\right)$ and by (25) we obtain

$$
\mathcal{L}\left(\theta_{n_{k}-1}, \theta_{m_{k}-1}, \varsigma_{0}\right) \quad<\mathcal{L}\left(\theta_{n_{k}}, \theta_{m_{k}}, \varsigma_{0}\right)
$$

From (22), (24), and (26), it follows that

$$
\begin{aligned}
1-\epsilon & \geq \mathcal{L}\left(\theta_{n_{k}}, \theta_{m_{k}}, \varsigma_{0}\right) \\
& >\mathcal{L}\left(\theta_{n_{k}-1}, \theta_{m_{k}-1}, \varsigma_{0}\right) \\
& \geq \mathcal{L}\left(\theta_{n_{k}-1}, \theta_{n_{k}}, \frac{\varsigma_{0}}{2}\right) * \mathcal{L}\left(\theta_{n_{k}}, \theta_{m_{k}-1}, \frac{\varsigma_{0}}{2}\right) \\
& >\mathcal{L}\left(\theta_{n_{k}-1}, \theta_{n_{k}}, \frac{\varsigma_{0}}{2}\right) *(1-\epsilon) .
\end{aligned}
$$

Letting $k \rightarrow+\infty$ and applying (21), we get 


$$
\lim _{k \rightarrow \infty} \mathcal{L}\left(\theta_{n_{k}-1}, \theta_{m_{k}-1}, \varsigma_{0}\right)=1-\epsilon=\lim _{k \rightarrow \infty} \mathcal{L}\left(\theta_{n_{k}}, \theta_{m_{k}}, \varsigma_{0}\right) .
$$

Taking $s_{k}=\mathcal{L}\left(\theta_{n_{k}-1}, \theta_{m_{k}-1}, \varsigma_{0}\right)$ and $t_{k}=\mathcal{L}\left(\theta_{m_{k}}, \theta_{n_{k}}, \varsigma_{0}\right)$, then $\lim _{k \rightarrow \infty} s_{k}=\lim _{k \rightarrow \infty} t_{k}=$ $1-\epsilon$. Applying condition $(\zeta 3)$, we get

$$
0 \leq \lim _{k \rightarrow \infty} \sup \zeta\left(\mathcal{L}\left(\theta_{m_{k}}, \theta_{n_{k}}, \varsigma_{0}\right), \mathcal{L}\left(\theta_{n_{k}-1}, \theta_{m_{k}-1}, \varsigma_{0}\right)\right)<0,
$$

a contradiction. As a result, $\left\{\theta_{n}\right\}$ is a Cauchy sequence. Since $(\mathcal{S}, \mathcal{L}, *)$ is a complete FMS, there exists $\theta^{*} \in \mathcal{S}$ such that

$$
\lim _{n \rightarrow+\infty} \mathcal{L}\left(\theta_{n}, \theta^{*}, \varsigma\right)=1
$$

From condition (iii), $\left\{\theta_{n}\right\}$ is a sequence in $\mathcal{S}$ such that $\alpha\left(\theta_{n}, \theta_{n+1}, \varsigma\right) \geq \eta\left(\theta_{n}, \theta_{n+1}, \varsigma\right)$ for all $n \in \mathbb{N}, \varsigma>0$ and $\theta_{n} \rightarrow \theta^{*} \in \mathcal{S}$. Hence, there exists a subsequence $\left\{\theta_{n(k)}\right\}$ of $\left\{\theta_{n}\right\}$ such that $\alpha\left(\theta_{n(k)}, \theta^{*}, \varsigma\right) \geq \eta\left(\theta_{n(k)}, \theta^{*}, \varsigma\right)$ and $\alpha\left(\theta^{*}, \mathcal{G} \theta^{*}, \varsigma\right) \geq \eta\left(\theta^{*}, \mathcal{G} \theta^{*}, \varsigma\right)$ for all $k \in \mathbb{N}, \varsigma>0$. Hence, we have

$$
\alpha\left(\theta_{n(k)}, \mathcal{G} \theta_{n(k)}, \varsigma\right) \alpha\left(\theta^{*}, \mathcal{G} \theta^{*}, \varsigma\right) \geq \eta\left(\theta_{n(k)}, \mathcal{G} \theta_{n(k)}, \varsigma\right) \eta\left(\theta^{*}, \mathcal{G} \theta^{*}, \varsigma\right) .
$$

From the definition of modified $\alpha-\eta-\mathcal{F} \mathcal{Z}$-contraction with respect to $\zeta \in \mathcal{F} \mathcal{Z}$, we have

$$
\begin{aligned}
0 & \leq \zeta\left(\mathcal{L}\left(\mathcal{G} \theta_{n(k)}, \mathcal{G} \theta^{*}, \varsigma\right), \mathcal{K}\left(\theta_{n(k)}, \theta^{*}, \varsigma\right)\right) \\
& =\zeta\left(\mathcal{L}\left(\theta_{n(k)+1}, \mathcal{G} \theta^{*}, \varsigma\right), \mathcal{K}\left(\theta_{n(k)}, \theta^{*}, \varsigma\right)\right) \\
& <\frac{1}{\mathcal{K}\left(\theta_{n(k)}, \theta^{*}, \varsigma\right)}-\frac{1}{\mathcal{L}\left(\theta_{n(k)+1}, \mathcal{G} \theta^{*}, \varsigma\right)},
\end{aligned}
$$

which yields

$$
\mathcal{K}\left(\theta_{n(k)}, \theta^{*}, \varsigma\right)<\mathcal{L}\left(\theta_{n(k)+1}, \mathcal{G} \theta^{*}, \varsigma\right),
$$

where

$$
\begin{aligned}
\mathcal{K}\left(\theta_{n(k)}, \theta^{*}, \varsigma\right) & =\min \left\{\mathcal{L}\left(\theta_{n(k)}, \theta^{*}, \varsigma\right), \max \left\{\mathcal{L}\left(\theta_{n(k)}, \mathcal{G} \theta_{n(k)}, \varsigma\right), \mathcal{L}\left(\theta^{*}, \mathcal{G} \theta^{*}, \varsigma\right)\right\}\right\} \\
& =\min \left\{\mathcal{L}\left(\theta_{n(k)}, \theta^{*}, \varsigma\right), \max \left\{\mathcal{L}\left(\theta_{n(k)}, \theta_{n(k)+1}, \varsigma\right), \mathcal{L}\left(\theta^{*}, \mathcal{G} \theta^{*}, \varsigma\right)\right\}\right\}
\end{aligned}
$$

Letting $k \rightarrow \infty$ in (28), we get

$$
\lim _{k \rightarrow \infty} \mathcal{K}\left(\theta_{n(k)}, \theta^{*}, \varsigma\right)=1 \leq \mathcal{L}\left(\theta^{*}, \mathcal{G} \theta^{*}, \varsigma\right)
$$

Therefore, $\mathcal{L}\left(\theta^{*}, \mathcal{G} \theta^{*}, \varsigma\right)=1$, thus $\mathcal{G} \theta^{*}=\theta^{*}$. This completes the proof.

By defining $\alpha(\theta, \vartheta, \varsigma)=1$ for all $\theta, \vartheta \in \mathcal{S}$ and all $\varsigma>0$, we conclude the following result:

Corollary 5. Let $(\mathcal{S}, \mathcal{L}, *)$ be a complete $F M S, \mathcal{G}: \mathcal{S} \longrightarrow \mathcal{S}$ be a $\eta$-subadmissible mapping satisfying the following conditions:

$$
1 \geq \eta(\theta, \mathcal{G} \theta, \varsigma) \eta(\vartheta, \mathcal{G} \vartheta, \varsigma) \Rightarrow \zeta(\mathcal{L}(\mathcal{G} \theta, \mathcal{G} \vartheta, \varsigma), \mathcal{K}(\theta, \vartheta, \varsigma)) \geq 0
$$

where $\mathcal{K}(\theta, \vartheta, \varsigma)=\min \{\mathcal{L}(\theta, \vartheta, \varsigma), \max \{\mathcal{L}(\theta, \mathcal{G} \theta, \varsigma), \mathcal{L}(\vartheta, \mathcal{G} \vartheta, \varsigma)\}\}$.

(i) there exists $\theta_{0} \in \mathcal{S}$ such that $1 \geq \eta\left(\theta_{0}, \mathcal{G} \theta_{0}, \varsigma\right)$; 
(ii) if $\left\{\theta_{n}\right\}$ is a sequence in $\mathcal{S}$ with $1 \geq \eta\left(\theta_{n}, \theta_{n+1}, \varsigma\right)$ for all $n \in \mathbb{N}, \varsigma>0$ and $\theta_{n} \rightarrow \theta \in \mathcal{S}$ as $n \rightarrow \infty$, then there exists a subsequence $\left\{\theta_{n(k)}\right\}$ of $\left\{\theta_{n}\right\}$ such that $1 \geq \eta\left(\theta_{n(k)}, \theta, \varsigma\right)$ and $1 \geq \eta(\theta, \mathcal{G} \theta, \varsigma)$ for all $k \in \mathbb{N}, \varsigma>0$.

Then $\mathcal{G}$ has a fixed point.

Example 4. Let $\mathcal{S}=[0, \infty)$ endowed with the fuzzy metric $\mathcal{L}: \mathcal{S} \times \mathcal{S} \times(0, \infty) \longrightarrow[0,1)$ given by $\mathcal{L}(\theta, \vartheta, \varsigma)=e^{-\frac{|\theta-\vartheta|}{\varsigma}}$ for all $\theta, \vartheta \in \mathcal{S}, \varsigma>0$ and $*$ be a product $t$-norm. We define $\mathcal{G}: \mathcal{S} \longrightarrow \mathcal{S}$ by

$$
\mathcal{G} \theta=\left\{\begin{array}{lc}
\frac{\theta^{2}}{4} & \text { if } \theta, \vartheta \in[0,1], \\
3 \theta^{2}+1 & \text { otherwise, }
\end{array}\right.
$$

and $\alpha, \eta: \mathcal{S} \times \mathcal{S} \times(0, \infty) \longrightarrow[0, \infty)$ by $\alpha(\theta, \vartheta, \varsigma)=1$,

$$
\eta(\theta, \vartheta, \varsigma)=\left\{\begin{array}{cc}
\frac{1}{4} & \text { if } \theta, \vartheta \in[0,1] \\
1 & \text { otherwise. }
\end{array}\right.
$$

We define $\zeta:(0,1] \times(0,1] \longrightarrow \mathbb{R}$ by $\zeta(\omega, \omega)=\frac{1}{\sqrt{\omega}}-\frac{1}{\omega}$. Let $(\theta, \vartheta) \in \mathcal{S} \times \mathcal{S}$, from the definition of $\alpha$ and $\eta$, we have that $1 \geq \eta(\theta, \vartheta, \varsigma)$ for all $\varsigma>0$ if and only if $\theta, \vartheta \in[0,1]$. Suppose that $1 \geq \eta(\theta, \vartheta, \varsigma)$, then $\theta, \vartheta, \in[0,1]$, since we have for all $\theta \in[0,1]$, and $\mathcal{G} \theta \in[0,1]$ it follows that $1 \geq \eta(\mathcal{G} \theta, \mathcal{G} \vartheta, \varsigma)$. Hence $\mathcal{G}$ is $\eta$-subadmissible.

Clearly, for any $\theta_{0} \in[0,1]$ we have $1 \geq \eta\left(\theta_{0}, \mathcal{G} \theta_{0}, \varsigma\right)$ for all $\varsigma>0$.

Now, let $\left\{\theta_{n}\right\}$ be a sequence such that $1 \geq \eta\left(\theta_{n}, \theta_{n+1}, \varsigma\right)$ for all $n \in \mathbb{N}$ and $\theta_{n} \rightarrow \theta \in \mathcal{S}$ as $n \rightarrow \infty$, it follows that $\left\{\theta_{n}\right\} \subseteq[0,1]$ and then $\theta \in[0,1]$, which implies that $1 \geq \eta\left(\theta_{n}, \theta, \varsigma\right)$ for all $n \in \mathbb{N}$ and $s>0$.

Next, we have to prove that (3) is satisfied and $(\theta, \vartheta) \in \mathcal{S} \times \mathcal{S}$ such that $1 \geq \eta(\theta, \mathcal{G} \theta, \varsigma) \eta(\vartheta, \mathcal{G} \vartheta, \varsigma)$ for all $\varsigma>0$. From the definition of $\alpha$ and $\eta$, we have $\theta, \vartheta \in[0,1]$. On the other hand, since

$$
\begin{aligned}
\frac{1}{\mathcal{L}(\mathcal{G} \theta, \mathcal{G} \vartheta, \varsigma)} & =\frac{1}{e^{-\frac{|\mathcal{G} \theta-\mathcal{G} \vartheta|}{\varsigma}}} \\
& =\frac{1}{e^{-\frac{\left|\theta^{2}-\vartheta^{2}\right|}{4 \zeta}}} \\
& \leq \frac{1}{e^{-\frac{|\theta-\vartheta|}{2 \varsigma}}} \\
& \leq \frac{1}{\sqrt{\mathcal{L}(\theta, \vartheta, \varsigma)}}
\end{aligned}
$$

Hence $\zeta(\mathcal{L}(\mathcal{G} \theta, \mathcal{G} \vartheta, \varsigma), \mathcal{K}(\theta, \vartheta, \varsigma)) \geq 0$. As all conditions of Corollary (5) are fulfilled, then $\mathcal{G}$ has a fixed point, $\theta=0$ is a fixed point to $\mathcal{G}$.

Corollary 6. Let $(\mathcal{S}, \mathcal{L}, *)$ be a complete FMS, $\mathcal{G}: \mathcal{S} \longrightarrow \mathcal{S}$ and $\alpha, \eta: \mathcal{S} \times \mathcal{S} \times(0, \infty) \longrightarrow[0, \infty)$ are mappings. Assume that there exists a function $\psi \in \Psi$ such that for all $\theta, \vartheta \in \mathcal{S}, \varsigma>0$,

$$
\alpha(\theta, \mathcal{G} \theta, \varsigma) \alpha(\vartheta, \mathcal{G} \vartheta, \varsigma) \geq 1 \Rightarrow \mathcal{L}(\mathcal{G} \theta, \mathcal{G} \vartheta, \varsigma) \geq \psi(\mathcal{K}(\theta, \vartheta, \varsigma))
$$

along with the following conditions:

(i) $\mathcal{G}$ is triangular $\alpha$-orbital admissible;

(ii) there exists $\theta_{0} \in \mathcal{S}$ such that $\alpha\left(\theta_{0}, \mathcal{G} \theta_{0}, \varsigma\right) \geq \eta\left(\theta_{0}, \mathcal{G} \theta_{0}, \varsigma\right)$;

(iii) if $\left\{\theta_{n}\right\}$ is a sequence in $\mathcal{S}$ such that $\alpha\left(\theta_{n}, \theta_{n+1}, \varsigma\right) \geq \eta\left(\theta_{n}, \theta_{n+1}, \varsigma\right)$ for all $n \in \mathbb{N}, \varsigma>0$ and $\theta_{n} \rightarrow \theta \in \mathcal{S}$, then there exists a subsequence $\left\{\theta_{n(k)}\right\}$ of $\left\{\theta_{n}\right\}$ such that $\alpha\left(\theta_{n(k)}, \theta, \varsigma\right) \geq$ $\eta\left(\theta_{n(k)}, \theta, \varsigma\right)$ for all $k \in \mathbb{N}, \varsigma>0$.

Then $\mathcal{G}$ has a fixed point. 
Proof. The conclusion can be drawn from Theorem 4 by defining $\eta(\theta, \vartheta, \varsigma)=1$ and $\zeta:(0,1] \times(0,1] \longrightarrow \mathbb{R}$ by $\zeta(\omega, \omega)=\frac{1}{\psi(\omega)}-\frac{1}{\omega}$ for all $\omega, \omega \in(0,1]$.

\section{Conclusions}

In this study, we use the $\mathcal{F} \mathcal{Z}$-simulation function technique to the study of certain fixed point results in FMS. By introducing the notions of $\alpha-\eta-\mathcal{F} \mathcal{Z}$-contractions, we unify, extend, and enrich various classical types of fuzzy contractions provided in $[5,6,23,25]$. We must emphasize that by appropriately defining the $\mathcal{F} \mathcal{Z}$-simulation function $\zeta$ and the admissible mappings $\alpha$ and $\eta$, we can particularize and deduce a variety of different consequences of our main results. On the other hand, further work is required in this direction of research because it appears reasonable to investigate the existence and uniqueness of a coincidence point or common fixed point of two self-mappings in a more general framework, for instance, partially ordered fuzzy metric spaces and so on.

Author Contributions: Conceptualization, A.M., N.S., S.M. and M.Z.; formal analysis, A.M., S.M. and M.Z.; investigation, M.Z. and N.S.; writing-original draft preparation, A.M., N.S., S.M. and M.Z.; writing-review and editing, N.S. and M.Z. All authors have read and agreed to the published version of the manuscript.

Funding: This research received no external funding.

Data Availability Statement: Not applicable.

Conflicts of Interest: The authors declare no conflict of interest.

\section{References}

1. Mathews, G.S. Partial Metric Topology. Proc. 8th Summer Conference on General Topology and Applications. Ann. N. Y. Acad. Sci. 1994, 728, 183-197.

2. Romaguera, S.; Valero, O. A quantitative computational model for complete partial metric spaces via formal balls. Math. Struct. Comput. Sci. 2009, 19, 541-563. [CrossRef]

3. Border, K.C. Fixed Point Theorems with Applications to Economics and Game Theory; Cambridge University Press: New York, NY, USA, 1985.

4. Grabiec, M. Fixed points in fuzzy metric spaces. Fuzzy Sets Syst. 1988, 27, 385-389. [CrossRef]

5. Gregori, V.; Sapena, A. On fixed-point theorems in fuzzy metric spaces. Fuzzy Sets Syst. 2002, 125, 245-252 [CrossRef]

6. Miheţ, D. Fuzzy $\psi$-contractive mappings in non-archimedean fuzzy metric spaces. Fuzzy Sets Syst. 2008, 159, 739-744. [CrossRef]

7. Wardowski, D. Fuzzy contractive mappings and fixed points in fuzzy metric spaces. Fuzzy Sets Syst. 2013, 222, 108-114. [CrossRef]

8. Melliani, S.; Moussaoui, A. Fixed point theorem using a new class of fuzzy contractive mappings. J. Univers. Math. 2018, 1, 148-154.

9. Khojasteh, F.; Shukla, S.; Radenović, S. A new approach to the study of fixed point theory for simulation functions. Filomat 2015, 29, 1189-1194. [CrossRef]

10. Samet, B.; Vetro, C.; Vetro, P. Fixed point theorem for $\alpha-\psi$-contractive type mappings. Nonlinear Anal. 2012, 75, 2154-2165. [CrossRef]

11. Karapınar, E. Fixed points results via simulation functions. Filomat 2016, 30, 2343-2350. [CrossRef]

12. Phiangsungnoen, S.; Cho, Y.J.; Kumam, P. Fixed Point Results for Modified Various Contractions in Fuzzy Metric Spaces via $\alpha$-admissibl. Filomat 2016, 30, 1869-1881. [CrossRef]

13. Melliani, S.; Moussaoui, A.; Chadli, L.S. Recent Advances in Intuitionistic Fuzzy Logic Systems Theoretical Aspects and Applications: Fixed Point Theory, Contractive Mapping. Melliani, S., Castillo O., Eds.; In Fuzzy Metric Space in Studies in Fuzziness and Soft Computing; Springer: Geneva, Switzerland, 2019; Volume 372, pp. 273-282.

14. Mlaiki, N.; Özgür, N.Y.; Taş, N. New Fixed-Point Theorems on an S-metric Space via Simulation Functions. Mathematics 2019, 7,583. [CrossRef]

15. Hayel, N.S.; Khan, I.A.; Imdad, M.; Alfaqih, W.M. New fuzzy $\varphi$-fixed point results employing a new class of fuzzy contractive mappings. J. Intell. Fuzzy Syst. 2019, 37, 5391-5402.

16. Demma, M.; Saadati, R.; Vetro, P. Fixed point results on $b$ - metric space via Picard sequences and $b$-simulations. Iran. J. Math. Sci. Inform. 2016, 11, 123-136.

17. Argoubi, H.; Samet, S.; Vetro, C. Nonlinear contractions involving simulation functions in a metric space with a partial order. $J$. Nonlinear Sci. Appl. 2015, 8, 1082-1094. [CrossRef]

18. Zhou, M.; Saleem, N.; Liu, X.L.; Fulga, A.; Rolánd Lopéz de Hierro, A.F. A New Approach to Proinov-Type Fixed-Point Results in Non-Archimedean Fuzzy Metric Spaces. Mathematics 2021, 9, 3001. [CrossRef] 
19. Moussaoui, A.; Hussain, N.; Melliani, S. Global Optimal Solutions for Proximal Fuzzy Contractions Involving Control Functions. J. Math. 2021, 2021, 6269304. [CrossRef]

20. Popescu, O. Some new fixed point theorems for $\alpha$-Geraghty contraction type maps in metric spaces. Fixed Point Theory Appl. 2014, 2014, 190. [CrossRef]

21. Schweizer, B.; Sklar, A. Statistical metric spaces. Pac. J. Math. 1960, 10, 313-334. [CrossRef]

22. George, A.; Veeramani, P. On some results in fuzzy metric spaces. Fuzzy Sets Syst. 1994, 64, 395-399. [CrossRef]

23. Mishra, U.; Vetro, C.; Kumam, P. On modified $\alpha$ - $\phi$-fuzzy contractive mappings and an application to integral equations. J. Inequal. Appl. 2016, 2016, 67. [CrossRef]

24. Chuadchawna, P.; Kaewcharoen, A.; Plubtieng, S. Fixed point theorems for generalized $\alpha-\eta-\psi$-Geraghty contraction type mappings in $\alpha-\eta$-complete metric spaces. J. Nonlinear Sci. Appl. 2016, 9, 471-485. [CrossRef]

25. Gopal, D.; Vetro, C. Some new fixed point theorems in fuzzy metric spaces. Iran. J. Fuzzy Syst. 2014, 11, 95-107. [CrossRef]

26. Hayel, N.S.; Mohammad, I.; Idrees, K. Hasanuzzaman Md, Fuzzy $\Theta_{f}$-contractive Mappings and Their Fixed Points with Applications. J. Intell. Fuzzy Syst. 2020, 39, 7097-7106. 